\title{
SDSS-IV MaNGA: Inside-out versus Outside-in Quenching of Galaxies in Different Local Environments
}

\author{
Lihwai Lin ${ }^{1}\left(\mathbb{D}\right.$, Bau-Ching Hsieh ${ }^{1}$ (), Hsi-An Pan ${ }^{1} \mathbb{1}$, Sandro B. Rembold ${ }^{2,3}$, Sebastián F. Sánchez ${ }^{4}$, Maria Argudo-Fernández ${ }^{5,6}$, \\ Kate Rowlands $^{7}$, Francesco Belfiore ${ }^{8}$, Dmitry Bizyaev ${ }^{9,10}$ (D), Ivan Lacerna ${ }^{11,12}$ (D), Rogréio Riffel ${ }^{3,13}$, Yu Rong ${ }^{6,14,15}$ (iD), \\ Fangting Yuan ${ }^{16}$, Niv Drory ${ }^{17}$ (D), Roberto Maiolino ${ }^{18,19}$, and Eric Wilcots ${ }^{20}$ \\ ${ }^{1}$ Institute of Astronomy \& Astrophysics, Academia Sinica, Taipei 10617, Taiwan; lihwailin@asiaa.sinica.edu.tw \\ ${ }^{2}$ Departamento de Física, CCNE, Universidade Federal de Santa Maria, 97105-900, Santa Maria, RS, Brazil \\ ${ }^{3}$ Laboratório Interinstitucional de e-Astronomia, Rua General José Cristino, 77 Vasco da Gama, Rio de Janeiro, 20921-400, Brazil \\ ${ }^{4}$ Instituto de Astronomía, Universidad Nacional Autónoma de México, Circuito Exterior, Ciudad Universitaria, Ciudad de México 04510, Mexico \\ ${ }^{5}$ Centro de Astronomía, Universidad de Antofagasta, Avenida Angamos 601, Antofagasta 1270300, Chile \\ ${ }^{6}$ Chinese Academy of Sciences South America Center for Astronomy, China-Chile Joint Center for Astronomy, Camino El Observatorio, 1515, Las Condes, \\ Santiago, Chile \\ ${ }^{7}$ Department of Physics \& Astronomy, Johns Hopkins University, Bloomberg Center, 3400 N. Charles Street, Baltimore, MD 21218, USA \\ ${ }_{9}^{8}$ UCO/Lick Observatory, University of California, Santa Cruz, 1156 High Street, Santa Cruz, CA 95064, USA \\ ${ }^{9}$ Apache Point Observatory and New Mexico State University, P.O. Box 59, Sunspot, NM, 88349-0059, USA \\ ${ }^{10}$ Sternberg Astronomical Institute, Moscow State University, Moscow, Russia \\ ${ }^{11}$ Instituto de Astronomía, Universidad Católica del Norte, Av. Angamos 0610, Antofagasta, Chile \\ ${ }^{12}$ Instituto Milenio de Astrofísica, Av. Vicuña Mackenna 4860, Macul, Santiago, Chile
Departamento de Astronomia, Universidade Federal do Rio Grande do Sul—Av. Bento Gonçalves 9500, Porto Alegre, RS, Brazil \\ ${ }^{14}$ Instituto de Astrofísica, Pontificia Universidad Católica de Chile, Av. Vicuña Mackenna 4860, Macul, Santiago, Chile \\ ${ }^{15}$ Key Laboratory for Computational Astrophysics, National Astronomical Observatories, Chinese Academy of Sciences, 20A Datun Road, Chaoyang District, \\ Beijing 100012, People's Republic of China \\ ${ }^{16}$ Shanghai Astronomical Observatory, Chinese Academy of Science, 80 Nandan Road, Shanghai 200030, People's Republic of China \\ ${ }_{17}$ McDonald Observatory, University of Texas at Austin, University Station, Austin, TX 78712-0259, USA \\ ${ }^{18}$ Cavendish Laboratory, University of Cambridge, 19 J. J. Thomson Avenue, Cambridge CB3 OHE, UK \\ ${ }^{19}$ University of Cambridge, Kavli Institute for Cosmology, Cambridge, CB3 OHE, UK. \\ ${ }^{20}$ Department of Astronomy, University of Wisconsin-Madison, 475N. Charter Street, Madison WI 53703, USA \\ Received 2018 October 3; revised 2018 December 2; accepted 2018 December 20; published 2019 February 8
}

\begin{abstract}
The large Integral Field Spectroscopy surveys have allowed the classification of ionizing sources of emission lines on sub-kiloparsec scales. In this work, we define two non-parametric parameters, quiescence $\left(F_{q}\right)$ and its concentration $\left(C_{q}\right)$, to quantify the strength and the spatial distribution of the quenched areas, respectively, traced by the $\mathrm{LI}(\mathrm{N}) \mathrm{ER}$ regions with low EW(H $\alpha)$. With these two measurements, we classify MaNGA galaxies into inside-out and outside-in quenching types according to their locations on the $F_{q}$ versus $C_{q}$ plane and we measure the fraction of inside-out (outside-in) quenching galaxies as a function of halo mass. We find that the fraction of galaxies showing inside-out quenching increases with halo mass, irrespective of stellar mass or galaxy type (satellites versus centrals). In addition, high-stellar-mass galaxies exhibit a greater fraction of inside-out quenching compared to low-stellar-mass ones in all environments. In contrast, the fraction of outside-in quenching does not depend on halo mass. Our results suggest that morphological quenching may be responsible for the inside-out quenching seen in all environments. On the other hand, the flat dependence of the outside-in quenching on halo mass could be a mixed result of ram pressure stripping and galaxy mergers. Nevertheless, for a given environment and stellar mass, the fraction of inside-out quenching is systematically greater than that of outside-in quenching, suggesting that inside-out quenching is the dominant quenching mode in all environments.
\end{abstract}

Key words: galaxies: evolution - galaxies: general - galaxies: star formation

\section{Introduction}

It has been long recognized that environments play an important role in galaxy evolution (Dressler 1980; Baldry et al. 2006; Cooper et al. 2007; Peng et al. 2010; Muzzin et al. 2012; Wetzel et al. 2012). Galaxies located in dense environments may experience short periods of mass assembly where different processes of star formation quenching are present such that their stellar populations are in general old with high metallicities, compared to those of field galaxies (e.g., Roberts \& Haynes 1994; Kauffmann 1996; Kuntschner et al. 2002; Trager et al. 2008; Blanton \& Moustakas 2009).

Studies of the main-sequence galaxies in various environments find that the specific star formation rate (sSFR) of galaxies located in groups or clusters is systematically lower by $0.1-0.3 \mathrm{dex}$ when compared to that of field galaxies
(Vulcani et al. 2010; Haines et al. 2013; Lin et al. 2014; Jian et al. 2017, 2018). Nevertheless, recent works have concluded that the well-established "color-density" relation at low redshifts is primarily driven by the increase of the quiescent population, with the global reduction in the star formation rate of star-forming galaxies in dense environments only being a secondary effect (Baldry et al. 2004; Koyama et al. 2013; Lin et al. 2014; Lacerna et al. 2016, 2018; Jian et al. 2017; ArgudoFernández et al. 2018). Combining all these results, it is suggested that there could be a mixing of various quenching processes that operate on different timescales going on for group/cluster galaxies.

A number of environment-associated mechanisms have been proposed to explain the suppressed star formation rate in dense environments. For example, the gas in galaxy disks or halos 
can be stripped when galaxies fall into a cluster and move through the hot intracluster medium, often referred to as ram pressure stripping (Gunn \& Gott 1972; McCarthy et al. 2008). This scenario is supported by the depleted H I gas and offset ionized gas observed in low-redshift cluster galaxies (e.g., Brown et al. 2017; Fritz et al. 2017). Similarly, the outer hot halo of galaxies may also be removed due to tidal forces or ram pressure, referred to as "strangulation," in which galaxies lose their fuel for further star formation (Larson et al. 1980). Other processes, such as galaxy interactions or galaxy harassment (Moore et al. 1996), which are found more frequently in dense environments (Lin et al. 2010), may also induce gas inflow toward the centers of galaxies, trigger starbursts, and consume the gas completely (Mihos \& Hernquist 1994; Cox et al. 2006). While it is likely that multiple processes contribute to the lower levels of star formation activity in dense environments, it is observationally very challenging to identify which mechanism is dominant over others.

Recent integral field spectroscopy (IFS) surveys, such as CALIFA (Sánchez et al. 2012), SAMI (Bryant et al. 2015), and MaNGA (Bundy et al. 2015), provide great opportunities to probe the quenching effect through spatially resolved information, as different quenching processes may leave distinct imprints on the spatial distributions of the star formation. For processes like ram pressure stripping or strangulation, one expects that the gas suppression and star formation quenching happen outside-in or globally, while AGN feedback would result in the opposite trend (inside-out). With the resolved information, it is therefore possible to constrain the quenching mechanisms by investigating the spatial patterns of quenching within the galaxies (e.g., González Delgado et al. 2014, 2016; Li et al. 2015; Tacchella et al. 2015; Lin et al. 2017a; Ellison et al. 2018; Sánchez et al. 2018), including their dependence on the stellar mass, morphology, and environment (Pérez et al. 2013; González Delgado et al. 2015, 2017; Pan et al. 2015; Ibarra-Medel et al. 2016; Schaefer et al. 2017; Medling et al. 2018; Sánchez et al. 2018; Spindler et al. 2018; Wang et al. 2018). Depending on the tracers used to study the quenching, some are more sensitive to the instantaneous halt of star formation (e.g., star formation rate), while others may in fact probe the aging effect (e.g., age, D4000 strength, etc.).

The environmental dependencies of resolved star formation activities so far have yielded controversial results. Schaefer et al. (2017) adopted the nearest neighbor local density as a environment tracer and studied the star formation rate gradients in the SAMI sample. They concluded that the star formation quenching occurs outside-in in dense environments. On the other hand, Spindler et al. (2018) utilized galaxies taken from the MaNGA survey and found a global suppression in the star formation rate from inner to outer regions for satellite galaxies, which favors the strangulation scenario.

In this paper, we investigate the spatial pattern of quenching and its dependence on the local environment, specifically the halo mass, in the MaNGA sample by quantifying the spatial distribution of quenched areas using non-parametric methods. We study the fractions of galaxies showing inside-out and outside-in quenching features as a function of halo mass for central and satellites galaxies separately, from which we infer the environment quenching mechanisms that operate in massive halos.

Throughout this paper we adopt the following cosmology: $H_{0}=100 h \mathrm{~km} \mathrm{~s}^{-1} \mathrm{Mpc}^{-1}, \Omega_{\mathrm{m}}=0.3$ and $\Omega_{\Lambda}=0.7$. We use a
Salpeter IMF and adopt the Hubble constant $h=0.7$. All magnitudes are given in the $\mathrm{AB}$ system.

\section{Data \\ 2.1. MaNGA IFU Data}

MaNGA (Bundy et al. 2015; Yan et al. 2016a, 2016b; Wake et al. 2017) is an integral field unit (IFU) survey on the SDSS $2.5 \mathrm{~m}$ telescope (Gunn et al. 2006), as part of the SDSS-IV survey (Albareti et al. 2017; Blanton et al. 2017). MaNGA makes use of a modification of the BOSS spectrographs (Smee et al. 2013) to bundle fibers into hexagons (Drory et al. 2015). Each spectrum has a wavelength coverage of 3500-10,000 and instrumental resolution $\sim 60 \mathrm{~km} \mathrm{~s}^{-1}$. After dithering, MaNGA data have an effective spatial resolution of 2.15 (FWHM; Law et al. 2015), and datacubes are gridded with $0 . " 5$ spaxels.

In this study, we use $\sim 4690$ galaxies with $z<0.15$ taken from the MaNGA MPL- 6 version of the internal release. To eliminate the effect of inclination on our analysis, we only use galaxies with a major-to-minor-axis ratio (b/a) greater than 0.4 (i.e., excluding high-inclination systems with $i>68^{\circ}$ ). This selection results in 4273 galaxies in our sample. We make use of the Pipe3D pipeline (Sánchez et al. 2016a) to model the stellar continuum with the GSD156 library of simple stellar populations (SSPs; Cid Fernandes et al. 2013) that comprises 156 templates covering 39 stellar ages (from $1 \mathrm{Myr}$ to $14.1 \mathrm{Gyr})$, and 4 metallicities $(Z / Z \odot=0.2,0.4,1$, and 1.5$)$, extracted from a combination of the synthetic stellar spectra from the GRANADA library (Martins et al. 2005) and the MILES project (Sánchez-Blázquez et al. 2006; Vazdekis et al. 2010; Falcón-Barroso et al. 2011). Details of the fitting procedures are described in Sánchez et al. (2016b). In short, a spatial binning is first performed in order to reach a signal-tonoise ratio $(\mathrm{S} / \mathrm{N})$ of 50 across the entire field of view $(\mathrm{FoV})$ for each datacube. A stellar population fit of the coadded spectra within each spatial bin is then computed. The stellar population model for spaxels with continuum $\mathrm{S} / \mathrm{N}>3$ is then estimated by rescaling the best fitted model within each spatial bin to the continuum flux intensity in the corresponding spaxel, following Cid Fernandes et al. (2013) and Sánchez et al. (2016a). The stellar-mass surface density $\left(\Sigma_{*}\right)$ is obtained using the stellar mass derived for each spaxel and normalized to the physical area of one spaxel. The best-fit stellar continuum is then subtracted from the reduced data spectrum for the emission-line measurements, which are measured spaxel by spaxel using a weighted momentum analysis as described in Sánchez et al. (2016b, 2018). All the emission lines were dust-extinctioncorrected using the Balmer decrement computed at each spaxel of the IFU cube, following the method described in the Appendix of Vogt et al. (2013). An extinction law with $\mathrm{Rv}=4.5 \quad$ (Fischera \& Dopita 2005) and Calzetti (2001) attenuation curve is used. These emission-line measurements are later used for the ionizing source classification through the Baldwin-Phillips-Terlevich (BPT) excitation diagnostic diagrams (Baldwin et al. 1981; Veilleux \& Osterbrock 1987; Kauffmann et al. 2003; Kewley et al. 2006). The classification is not sensitive to the dust reddening law since the lines in each pair used for the line ratio calculations are close enough in wavelength. 


\subsection{Halo Mass}

The halo masses of our sample are adopted from the group catalog kindly made available by X. Yang et al. (2018, in private communication). This catalog updates the SDSS DR4 group catalog of Yang et al. $(2007,2008)$ to the SDSS DR7 version. The galaxy groups are identified using an adaptive halo-based group finder based on the NYU-VAGC Catalog (Blanton et al. 2005). The match to Yang's group catalog results in a subsample of 2915 galaxies with halo mass measurements.

For each galaxy, the group catalog provides two estimates for its halo mass: (1) $M_{L}$, based on the ranking of the characteristic luminosity $L_{19.5}$, the total luminosity of all group members with ${ }^{0.1} M_{r}-5 \log h<=19.5$; (2) $M_{s}$, based on the ranking of the characteristic stellar mass $M_{\text {stel }}$, the total stellar mass of all group members with ${ }^{0.1} M_{r}-5 \log h<=19.5$. Detailed tests with mock catalogs have shown that the halo masses are estimated reliably, with a standard deviation of about 0.3 dex (Yang et al. 2008). The two halo mass estimates yield very similar results for our analyses. In this paper, we present the results based on the luminosity-based halo mass $M_{L}$.

\section{Methods}

With the advent of large IFU surveys, such as CALIFA, MaNGA, etc., it is now possible to probe the emission-line properties in subregions within the galaxies. In Hsieh et al. (2017), we confirmed the so-called "resolved star-forming main sequence" (Sánchez et al. 2013; Cano-Díaz et al. 2016; González Delgado et al. 2016), the tight correlation between the star formation rate surface density and the stellar-mass surface density $\left(\Sigma_{*}\right)$, on kiloparsec scales for $\mathrm{H}$ II regions classified based on the BPT line diagnostics. In addition, we found that the $\mathrm{H} \alpha$ surface density $\left(\Sigma_{\mathrm{H} \alpha}\right)$ is also strongly correlated with $\Sigma_{*}$ for regions classified as LI(N)ERs. The emission power of $\mathrm{LI}(\mathrm{N}) \mathrm{ER}$ regions is lower than that of the $\mathrm{H}$ II regions by nearly two orders of magnitude at a fixed $\Sigma_{*}$. The existence of this relation is in support of the scenario that LI(N)ER regions are primarily powered by hot evolved stars (e.g., Binette et al. 1994; Stasińska et al. 2008; Sarzi et al. 2010; Yan \& Blanton 2012; Papaderos et al. 2013; Singh et al. 2013; Belfiore et al. 2016, 2017; Gomes et al. 2016), whose abundance is proportional to the stellar mass. Since the emissions from hot evolved stars only begin to dominate after tens of Myr after the OB stars stop forming (Zhang et al. 2017) and the emission power of LI(N)ER is only a small percent of that of star-forming regions (Hsieh et al. 2017), spaxels classified as $\mathrm{LI}(\mathrm{N}) \mathrm{ER}$ can be regarded as regions where the star formation has already ceased. This kind of association between the LI(N)ER regions and the "retired" or "quenched" areas has been previously investigated in other works (Singh et al. 2013; Belfiore et al. 2016, 2017). Nevertheless, it is worth noting that some LI(N)ER-like emission may be associated with a weakly active galactic nucleus or shocks (e.g., in interacting galaxies), which normally have high equivalent widths (EWs). In order to remove contributions from non-quenching origins of $\mathrm{LI}(\mathrm{N})$ ERs, we apply an EW ( $\mathrm{H} \alpha)$ cut when identifying the quenched areas (see the next paragraph).

For a given galaxy, we can then quantify the degree and the spatial distribution of the quenched areas by looking into the abundance and locations of the quenched spaxels. In this work, we thus define two quantities, $F_{q}$ and $C_{q}$, to describe the fraction and the concentration of the quenched areas within a galaxy, respectively. For each spaxel of MaNGA data, we first classify the emission-line regions using the BPT diagrams. We adopt the dividing curves suggested in the literature (e.g., Kewley et al. 2001; Kauffmann et al. 2003; Cid Fernandes et al. 2010) to separate various regions into H II, LI(N)ER, composite, and AGN regimes using the $[\mathrm{N} \mathrm{II}] / \mathrm{H} \alpha$ and $[\mathrm{O} \mathrm{III]} /$ $\mathrm{H} \beta$ ratio as illustrated in the Figure 7 of Lin et al. (2017b). To differentiate contributions between quenched regions (or sometimes called "retired regions") and other ionizing sources (e.g., weakly AGNs or shocks) in powering LI(N)ERs, we further apply an $\operatorname{EW}(\mathrm{H} \alpha)>-3 \AA$ (positive value for absorption) cut in $\mathrm{LI}(\mathrm{N}) \mathrm{ER}$ spaxels when identifying final quenched areas (Cid Fernandes et al. 2011; Hsieh et al. 2017). Releasing the equivalent width criterion to $\operatorname{EW}(\mathrm{H} \alpha)>-6 \AA$ (e.g., Sánchez et al. 2015) would result in an increase of the inside-out quenching fraction (defined below) by $\sim 10 \%$ and change the outside-in quenching fraction by differences ranging from $-50 \%$ to $+20 \%$. Nevertheless, this does not affect our main results and conclusions.

For each galaxy, we define the quenched fraction (or "quiescence" hereafter), $F_{q}$, as the following:

$$
F_{q}=N_{\text {quenched }} / N_{\text {all }} \text {, }
$$

where $N_{\text {all }}$ is the total number of spaxels within $1.5 R_{e}$ with stellar-mass surface density greater than $10^{6} M_{\odot} \mathrm{kpc}^{-2}$ and with at least one of the four emission lines $(\mathrm{H} \alpha, \mathrm{H} \beta,[\mathrm{N} \mathrm{II}]$, [O III]) above the $\mathrm{S} / \mathrm{N}$ threshold (3 for $\mathrm{H} \alpha$ and $\mathrm{H} \beta$; 2 for [N II] and [O III]), and $N_{\text {quenched }}$ is the number of quenched spaxels (only galaxies with $N_{\text {quenched }} \geqslant 3$ are considered in this work). The stellar-mass surface density cut is motivated by the data distribution on the star formation rate surface density versus the stellar-mass surface density plane of Abdurro'uf \& Masayuki (2018, see their Figure 7). Our conclusions remain the same even if a higher cut of $10^{7} M_{\odot} \mathrm{kpc}^{-2}$ is applied.

The concentration of the quenched spaxels (hereafter "quenching concentration"), $C_{q}$, is computed as:

$$
C_{q}=\sum r_{\text {all }}^{2} / \sum r_{\text {quenched }}^{2},
$$

where $r$ refers to the distance of a given spaxel to the galaxy center, corrected for the inclination. While $F_{q}$ represents the degree of quenching in a galaxy, $C_{q}$ reflects the spatial distribution of the quenched area.

The combination of $F_{q}$ and $C_{q}$ provides a powerful method for describing the spatial sequence of quenching. At a fixed $F_{q}$, galaxies with quenching occurring in the inner regions have a greater value of $C_{q}$ than those with quenching occurring in the outskirts. In other words, the inside-out quenching and outsidein quenching will follow different trajectories in the $F_{q}$ versus $C_{q}$ diagram. To illustrate this point, we perform two sets of toy models, one with inside-out quenching, starting from the inner spaxels to the outer spaxels (see the left sequence in Figure 1) and the other with outside-in quenching, proceeding with the opposite direction (see the middle sequence in Figure 1), assuming a perfect circle. For each set of models, we create 80 equally spaced annulus bins. In each step, we shift the annulus boundary that separates the quenched and unquenched areas by one annulus bin and compute $F_{q}$ and $C_{q}$. The obtained insideout and outside-in quenching trajectories are shown as the dotted-dashed line and the dotted line in Figure 2, respectively. In fact, these two trajectories can also be derived analytically. 


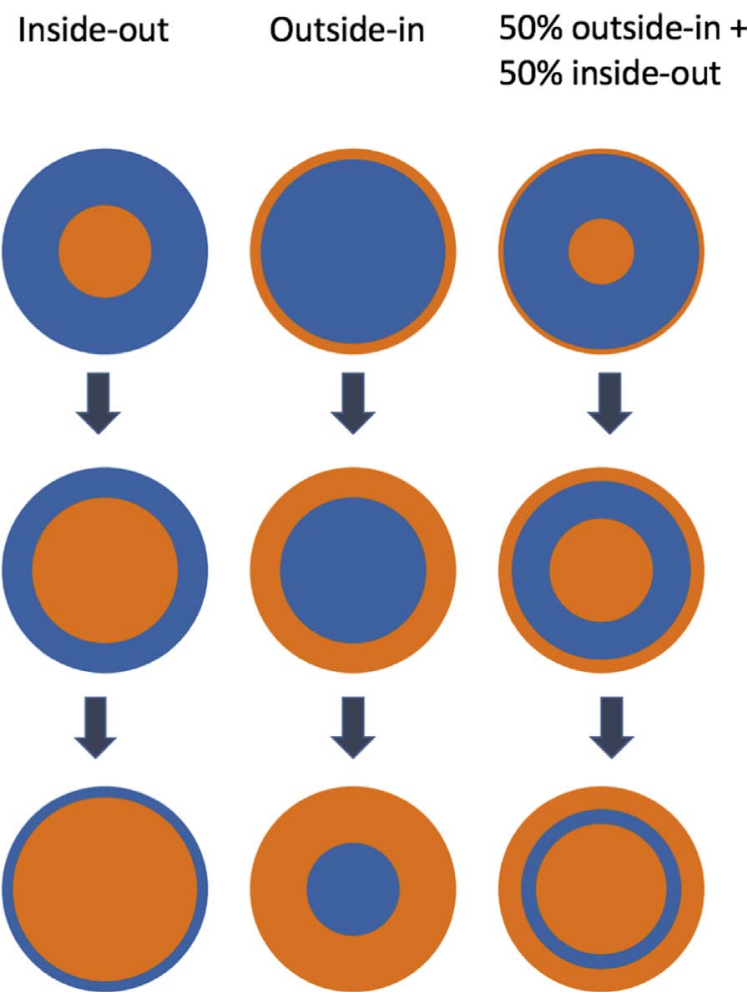

Figure 1. Schematic plot showing the time evolution of the quenching used in our toy models (see Section 3 and the Appendix). Regions with ongoing star formation are shown in blue, whereas regions where the star formation has ceased are shown in red. Left sequence: inside-out quenching-star formation is quenched in the center first and then proceeds outward. Middle sequence: outside-in quenching-quenching proceeds inwards from the outer parts of galaxies. Right sequence: a mixture of $50 \%$ inside-out quenching and $50 \%$ outside-in quenching.

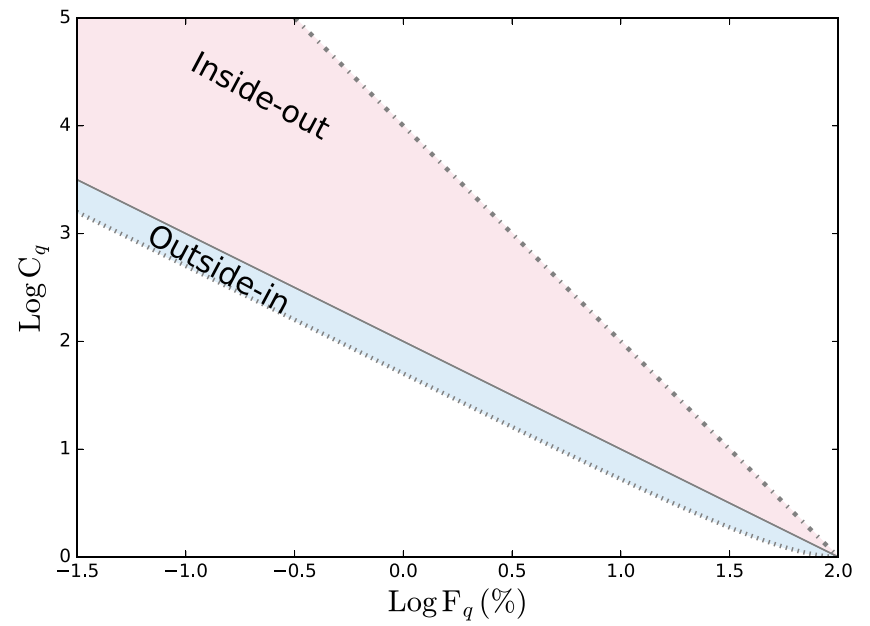

Figure 2. Distributions of two toy models on the quiescence $\left(F_{q}\right)$ vs. quenching concentration $\left(C_{q}\right)$ plane. The dotted line represents the outside-in quenching sequence whereas the dotted-dashed line shows the inside-out quenching sequence. The solid line is the dividing line that separates the inside-out-like (pink area) and outside-in-like (light blue area) quenching modes (see the text of Section 4.2 and the Appendix). (As the toy models assume circular geometry and might be too simplistic, it is inevitable that some galaxies may fall outside the model boundaries, in which case the galaxies are still categorized according to whether they lie above or below the dividing line.)

In the Appendix, we consider a general case where there is a mixture of inside-out and outside-in quenching within a galaxy (see the right sequence in Figure 1). The equations describing

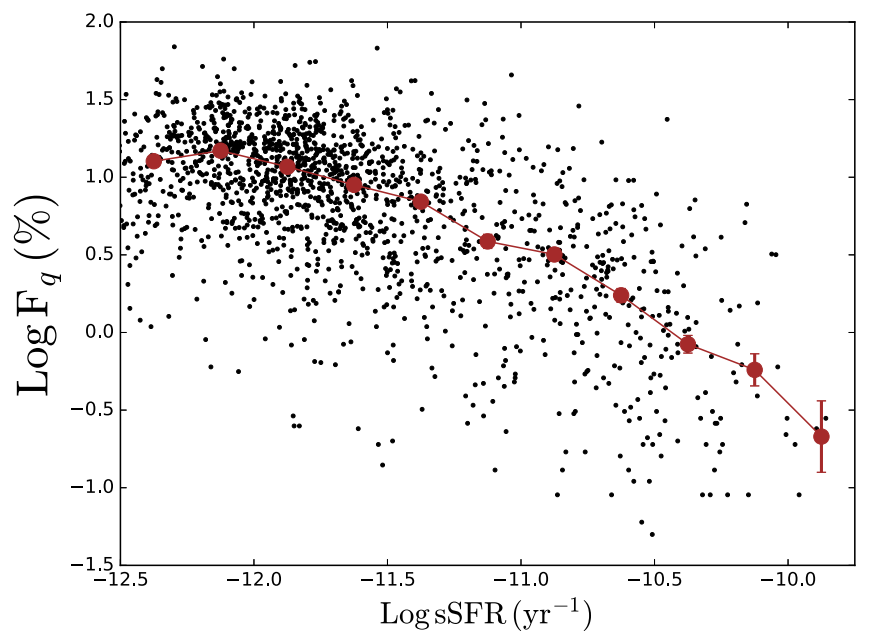

Figure 3. Quiescence $\left(F_{q}\right)$ vs. sSFR for the MaNGA sample. The black dots represent the measurements of each individual galaxy. The brown symbols show the medians and the associated uncertainties (computed as the root-meansquare in the logarithm space normalized by the square root of the sample size in each bin) of galaxies with measurable $F_{q}$.

the pure inside-out and outside-in quenching lines can be obtained using Equation (23) by adopting $F_{q I}$ (the contribution of inside-out quenching) $=100$ and 0 , respectively:

$$
\begin{gathered}
\log _{10} C_{q}=-2 \log _{10} F_{q}+4 \text { (Inside-out), } \\
\log _{10} C_{q}=\log _{10} \frac{1}{1-\left(1-F_{q} / 100\right)^{2}} \text { (Outside-in). }
\end{gathered}
$$

By comparing the locations of the observed $F_{q}$ and $C_{q}$ of galaxies with the models, we are able to categorize whether a galaxy contains more inside-out quenching or outside-in quenching. The criteria we adopted for the selection of quenched areas only concern the stellar-mass surface density, the line equivalent width, and the line ratios, all of which do not directly depend on the observation resolution. As the two parameters $\left(F_{q}\right.$ and $\left.C_{q}\right)$ deal with the relative quantities, our method is not that sensitive to the spatial resolution in the case where there are sufficient resolution elements. Nevertheless, it is worth noting that very compact regions of quenched areas could be missed in our data and can only be resolved with greater spatial resolutions. Higher-resolution observations would be required to investigate whether this is an important effect or not.

\section{Results}

\subsection{The Dependence of Quiescence $\left(\mathrm{F}_{\mathrm{q}}\right)$ and Concentration $\left(\mathrm{C}_{\mathrm{q}}\right)$ on the Global sSFR}

Our working assumption is that the regions showing $\mathrm{LI}(\mathrm{N})$ ER-like emissions and with low $\mathrm{EW}(\mathrm{H} \alpha)$ are essentially places where the star formation has already ceased. If this is true, one would expect more active star-forming galaxies to have lower $F_{q}$ and quiescent galaxies to have high $F_{q}$. This correlation is illustrated in Figure 3, where we plot the quiescence $\left(F_{q}\right)$ versus the global sSFR computed from Pipe3D. As expected, the quiescence parameter decreases with increasing global sSFR, although with large scatter. The scatter is greater for galaxies with high sSFR. This is because the strength of the sSFR may differ in H II spaxels among galaxies even at a given fixed fraction of LI(N)ER spaxels. Nevertheless, it is still encouraging 


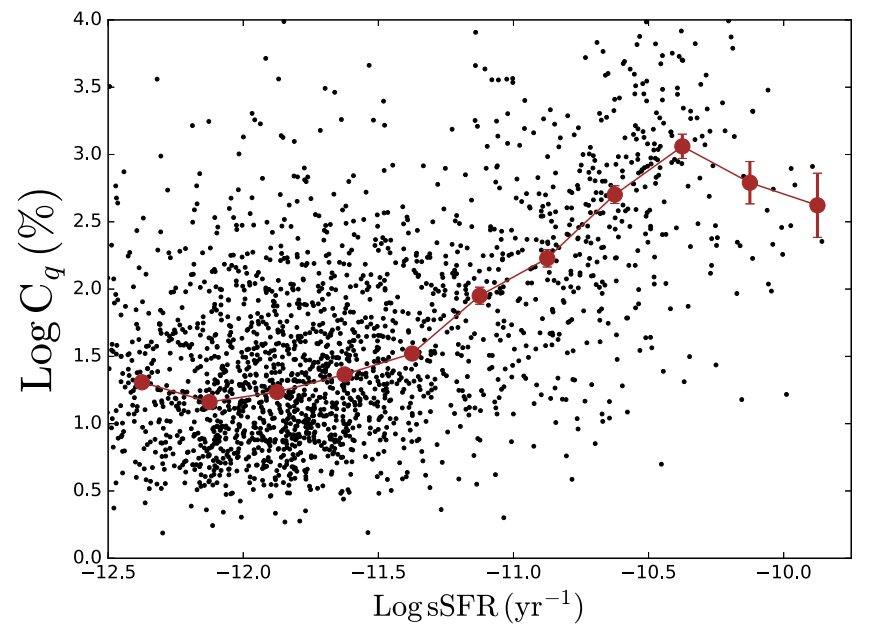

Figure 4. Concentration of quenched area $\left(C_{q}\right)$ vs. sSFR for the MaNGA sample. The black dots represent the measurements of each individual galaxy. The brown symbols show the medians and the associated uncertainties (computed as the root-mean-square in the logarithm space normalized by the square root of the sample size in each bin) of galaxies with measurable $C_{q}$.

to see the anticorrelation between the defined quiescence and global sSFR.

Next, we plot the quenching concentration $\left(C_{q}\right)$ as a function of global sSFR in Figure 4. We find that there is also a fairly good correlation between $C_{q}$ and global sSFR. Galaxies with lower sSFR tend to have more extended distributions of quenched area, and hence low $C_{q}$. However, we also note that $C_{q}$ seems to decline with sSFR for galaxies with $\log$ (sSFR) $>-10.5$. This is likely due to the small number statistics of both the sample size of galaxies in the two highest $\log (\mathrm{sSFR})$ bins and the small number of retired spaxels associated with them.

\subsection{Quiescence $\left(\mathrm{F}_{\mathrm{q}}\right)$ versus Quenching Concentration $\left(\mathrm{C}_{\mathrm{q}}\right)$}

In Figure 5 we plot the distributions of all MaNGA galaxies on the $F_{q}$ versus $C_{q}$ plane, color-coded according to their global sSFR. The two toy model lines are also shown to guide the eyes. It can be seen that our sample spreads over the regions between the two model lines on the $F_{q}$ versus $C_{q}$ plane. This can be attributed to two effects. First, the quenching may not occur subsequently with increasing or decreasing radius and/or there could be mixed modes of quenching for most of the galaxies. For example, when a centrally quenched galaxy due to prior AGN or morphological quenching falls into cluster environments and thus suffers from ram pressure stripping, it can exhibit both the inside-out and outside-in quenching features. Second, as illustrated in Figures 6 and 7, the spatial distribution of quenched areas may be patchy, not necessarily axisymmetric.

In order to properly classify galaxies into the outside-in and inside-out quenching categories, we analytically compute the $F_{q}$ and $C_{q}$ values by varying the relative contributions from the two modes and investigate how these two parameters change on the $F_{q}$ versus $C_{q}$ plane. The detailed calculations are given in the Appendix. As illustrated in Figure 14, even a small contribution from the outside-in quenching will move the locations toward the pure outside-in quenching line. Therefore, we define a dividing line (gray line) that corresponds to the $50 \%$ inside-out and $50 \%$ outside-in quenching contributions to separate the two types of quenching modes. Galaxies lying

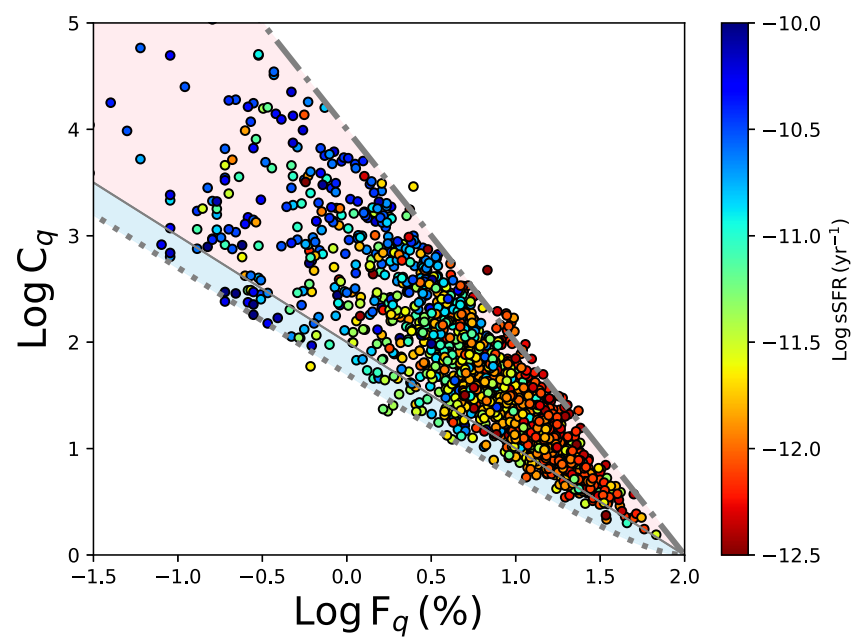

Figure 5. Distributions of MaNGA galaxies on the quiescence $\left(F_{q}\right)$ vs. quenching concentration $\left(C_{q}\right)$ plane, color-coded according to the their sSFR. Similar to Figure 2, the light blue shaded area denotes the outside-in-like quenching mode whereas the pink shaded region denotes the inside-out-like quenching mode.

below (above) this threshold line are counted as outside-in (inside-out) dominant quenching. Examples of galaxies classified as inside-out-like and outside-in-like quenching are shown in Figures 6 and 7, respectively.

Figure 8 presents the global SFR $-M_{*}$ distribution derived from the Pipe3D analysis of the full MaNGA sample, colorcoded according to their quenching types (red: inside-out; blue: outside-in; gray: unclassified). We split the sample into two stellar-mass bins. For massive galaxies $\left(M_{*}>10^{10.5} M_{\odot}\right)$, those objects showing either inside-out $(68 \%)$ or outside-in (5\%) quenching features are located in the lower side of the starforming sequence and the quiescent population. On the other hand, in the low-mass bin $\left(10^{9.5} M_{\odot}<M_{*}<10^{10.5} M_{\odot}\right), 31 \%$ are classified as inside-out quenching while $5 \%$ are classified as outside-in quenching. These low-mass galaxies with quenching features predominantly lie in the quiescent (passive) population.

\subsection{The Halo Mass Dependence of the Inside-out and Outside- in Quenching}

To see how environments might affect the quenching patterns of galaxies, we make a similar plot by binning the galaxies based on their hosting halo masses for all galaxies with $M_{*}>10^{9.5} M_{\odot}$ (top panels of Figure 9$)$. For this purpose, we limit our sample to 2915 galaxies that have halo mass measurements. As the strength of ram pressure stripping is proportional to the density of intergalactic medium (Gunn \& Gott 1972), it has been suggested to take place in denser environments, such as galaxy clusters. If ram pressure stripping is indeed a dominant process that quenches the star formation in cluster-like halos, we would expect to see fractionally more galaxies classified as outside-in quenching with increasing halo mass. As mentioned in Section 4.2, the fractions of galaxies that exhibit inside-out or outside-in quenching features are stellar-mass-dependent. In order to remove the stellar-mass effect, we also present the results in two stellar-mass bins, $10^{9.5} M_{\odot}<M_{*}<10^{10.5} M_{\odot}$ and $M_{*}>10^{10.5} M_{\odot}$, in the middle and bottom panels, respectively. The two stellar-mass cuts adopted here yield a similar dynamical range in terms of stellar mass and are also able to provide a sufficient number of satellites in different halo mass bins for subsequent analyses. 

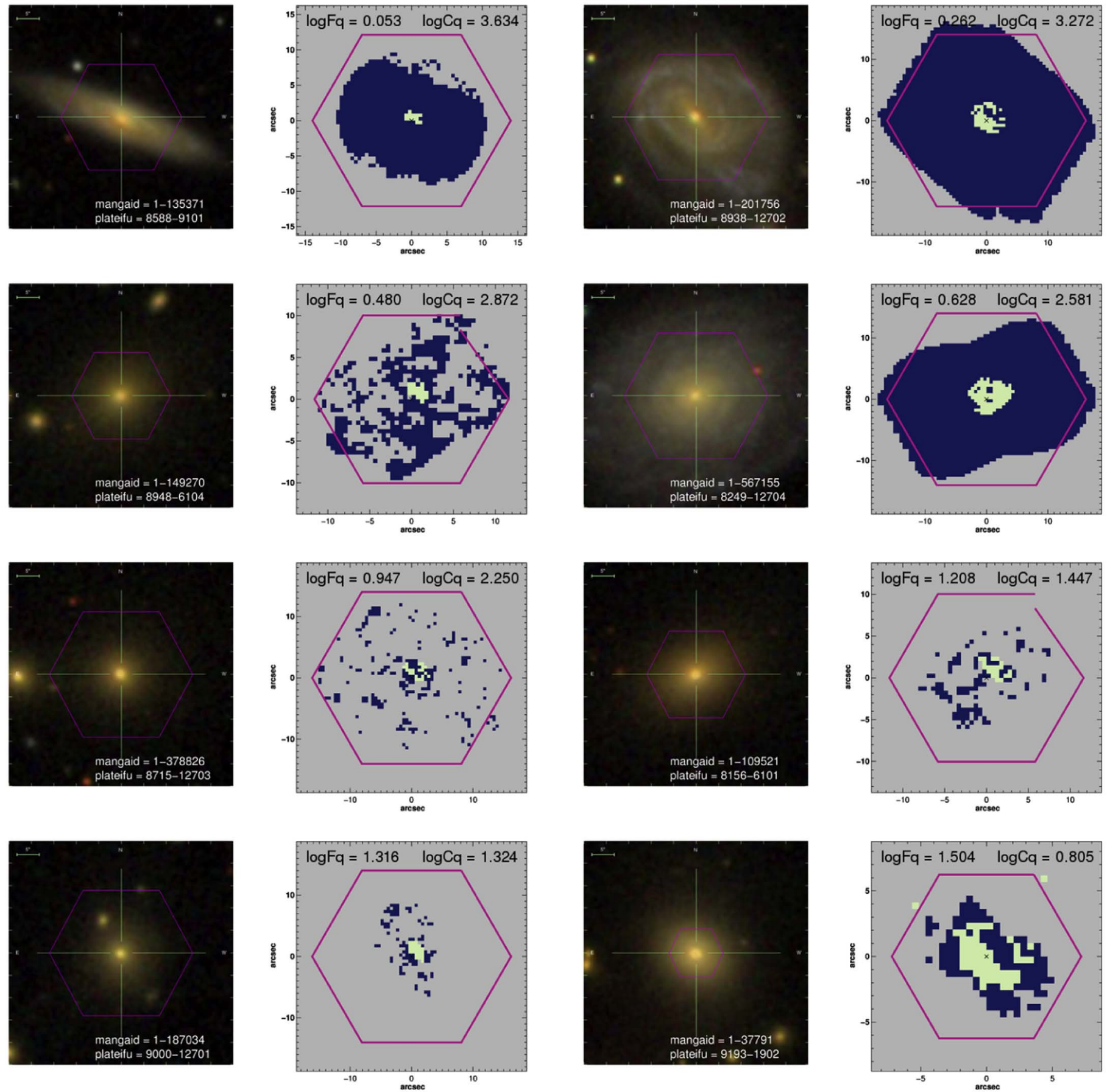

Figure 6. Examples of galaxies classified as inside-out-like quenching. The first and third columns show the SDSS gri composite images. The spatial distributions of the quenched areas are shown in yellow in the second and fourth columns, and are associated with the objects in the first and third columns, respectively. The dark blue marks the areas that satisfy the surface density of stellar mass and emission-line cuts described in Section 3.

For centrals and satellites, we compute the fraction of galaxies exhibiting inside-out quenching patterns $\left(f_{\text {in-out }}\right)$ as follows:

$$
\begin{aligned}
& f_{\text {in-out }}^{\text {cen. }}=N_{\text {in-out }}^{\text {cen. }} / N^{\text {cen }}, \\
& f_{\text {in-out }}^{\text {sat. }}=N_{\text {in-out }}^{\text {sat. }} / N^{\text {sat. }},
\end{aligned}
$$

where $N^{\text {cen. }}\left(N^{\text {sat. }}\right)$ is the number of total central (satellite) galaxies and $N_{\text {in-out }}^{\text {cen. }}\left(N_{\text {in-out }}^{\text {sat. }}\right)$ is the number of central (satellite) galaxies classified as inside-out quenching. There are galaxies, however, that do not have $\mathrm{LI}(\mathrm{N}) \mathrm{ER}$ spaxels within $1.5 R_{e}$, meaning that neither their $F_{q}$ nor $C_{q}$ is computed. These galaxies are typically star-forming galaxies, as illustrated in Figure 8, and cannot be categorized as either inside-out quenching or outside-in quenching. In other words, $N_{\text {in-out }}+$ $N_{\text {out-in }} \leqslant 1$. Therefore, we separately compute the fraction of galaxies exhibiting outside-in quenching patterns $\left(f_{\text {out-in }}\right)$ :

$$
\begin{aligned}
& f_{\text {out-in }}^{\text {cen. }}=N_{\text {out-in }}^{\text {cen. }} / N^{\text {cen }}, \\
& f_{\text {out-in }}^{\text {sat. }}=N_{\text {out-in }}^{\text {sat. }} / N^{\text {sat. }},
\end{aligned}
$$

where $N_{\text {out-in }}^{\text {cen. }}\left(N_{\text {out-in }}^{\text {sat. }}\right)$ is the number of central (satellite) galaxies classified as outside-in quenching. The derived values 

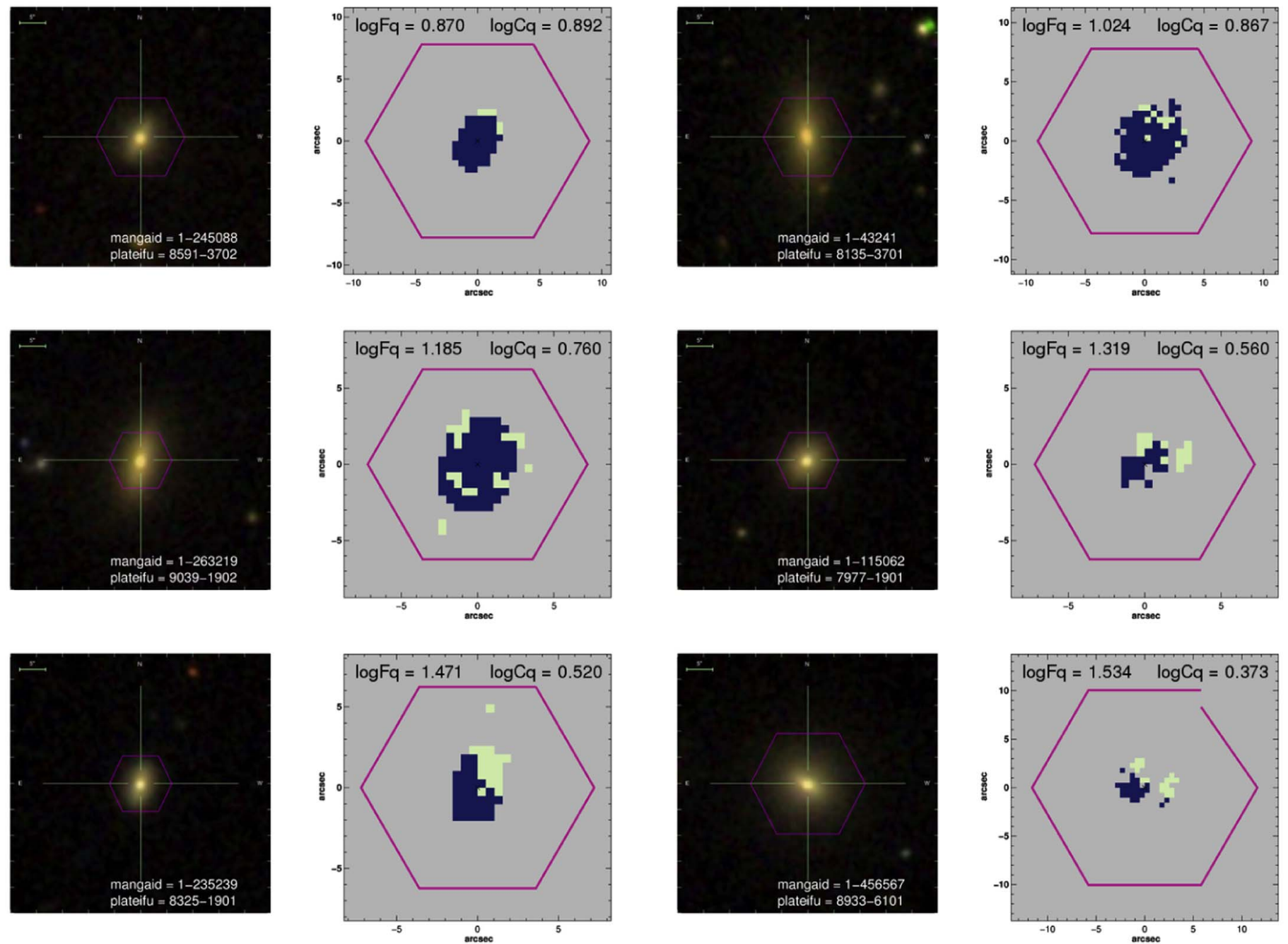

Figure 7. Examples of galaxies classified as outside-in-like quenching. The first and third columns show the SDSS gri composite images. The spatial distributions of the quenched areas are shown in yellow in the second and fourth columns, and are associated with the objects in the first and third columns, respectively. The dark blue marks the areas that satisfy the surface density of stellar mass and emission-line cuts described in Section 3.

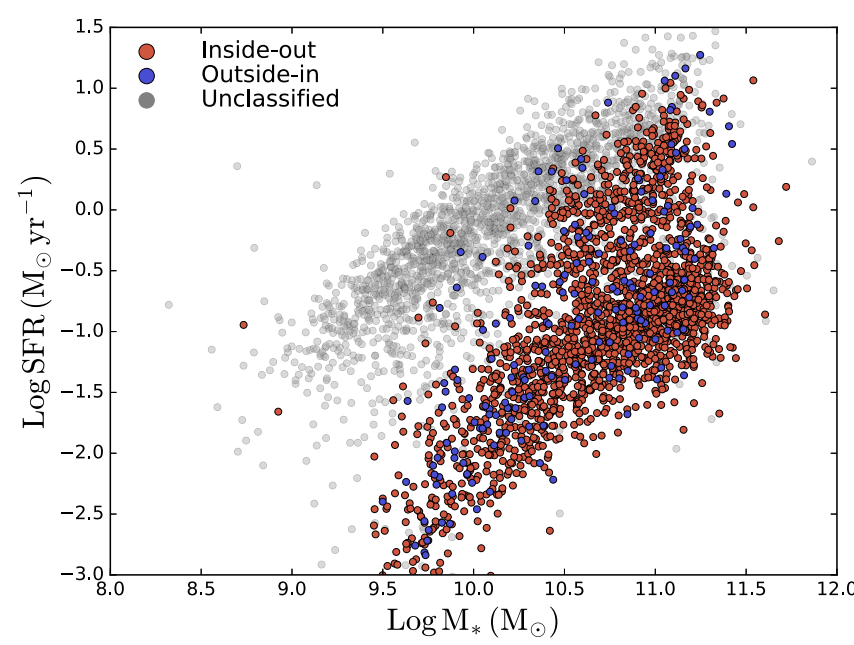

Figure 8. Distributions of MaNGA galaxies on the SFR vs. $M_{*}$ plane. The red and blue symbols denote galaxies classified as inside-out-like and outside-inlike quenching objects, respectively. Unclassified galaxies are shown as gray points. of $f_{\text {in-out }}$ and $f_{\text {out-in }}$ are shown in the upper right and lower left corners of each panel, respectively, as well as in Table 1.

In the left panel of Figure 10, we show the fraction of galaxies exhibiting inside-out quenching patterns $\left(f_{\text {in-out }}\right)$ as a function of halo mass. For centrals, we only present results with $M_{*}>10^{10.5} M_{\odot}$, as the statistics for less massive centrals are relatively poor in massive halos. We see that $f_{\text {in-out }}$ increases with hosting halo mass for both centrals and satellite galaxies. For the satellites, $f_{\text {in-out }}$ is significantly higher for high-stellarmass galaxies than the low-stellar-mass ones at a given halo mass. This phenomenon is consistent with the finding that the fraction of centrally suppressed galaxies or central LI(N)ER galaxies increases with the stellar mass (Belfiore et al. 2017; Spindler et al. 2018). Recalling that not all galaxies possess enough LI(N)ER features to be classified as inside-out quenching or outside-in quenching, $f_{\text {in-out }}$ and $f_{\text {out-in }}$ do not necessarily sum to one. To see the trend for the fraction of galaxies exhibiting outside-in quenching patterns $\left(f_{\text {out-in }}\right)$, we plot $f_{\text {out-in }}$ versus halo mass in the right panel of Figure 10 .

Interestingly, it is shown that the fraction of outside-in quenching galaxies does not strongly depend on the halo mass, 


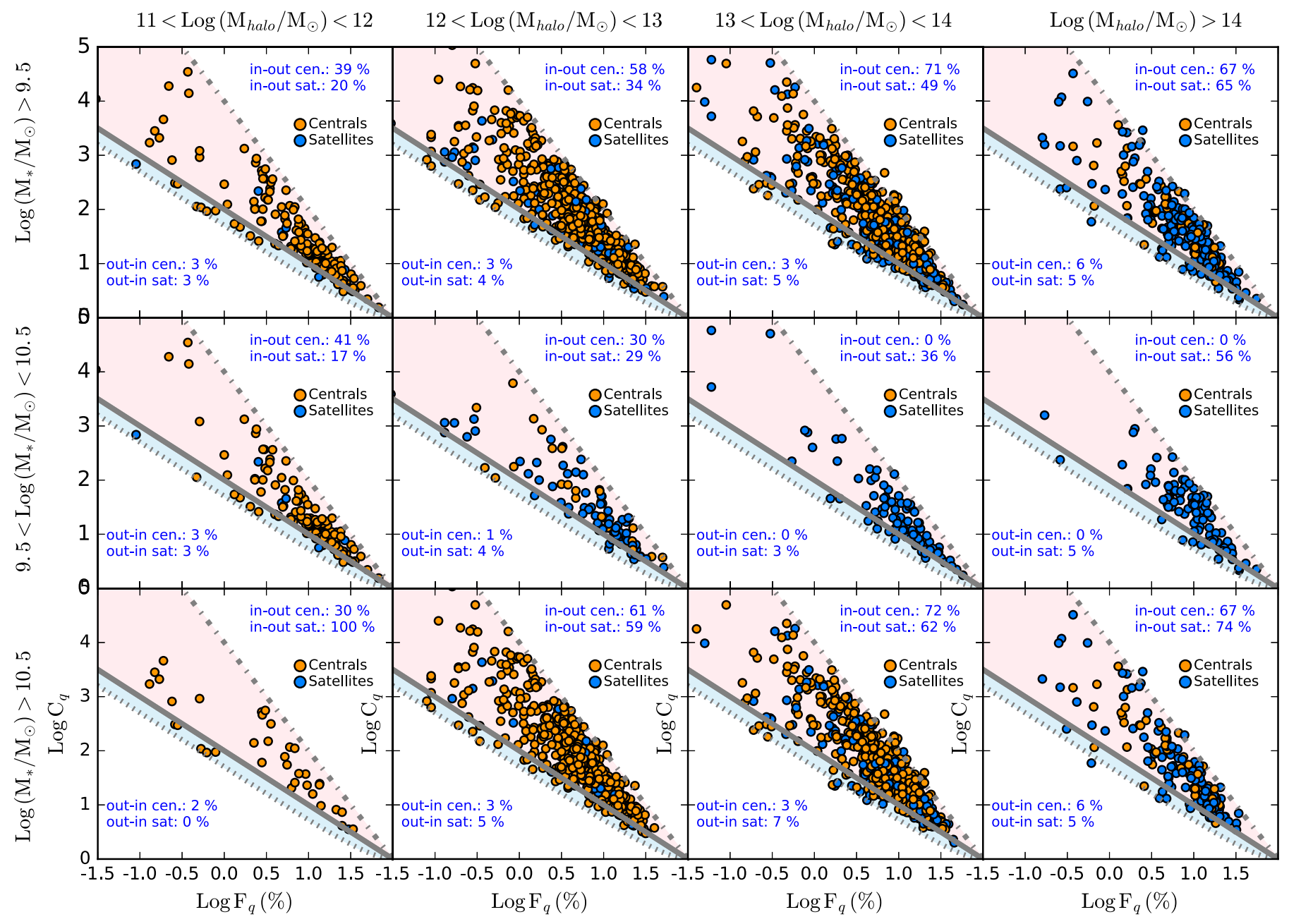

Figure 9. Distributions of all MaNGA galaxies on the quiescence $\left(F_{q}\right)$ vs. quenching concentration $\left(C_{q}\right)$ plane in different halo mass bins (increasing from left to right). The centrals and satellites are shown as orange and blue, respectively. Similar to Figure 2, the light blue shaded area denotes the outside-in-like quenching mode, whereas the pink shaded region denotes the inside-out-like quenching mode. The top, middle, and bottom panels are for galaxies with different stellar-mass cuts (from top to bottom: all galaxies with $M_{*}>10^{9.5} M_{\odot}, 10^{9.5} M_{\odot}<M_{*}<10^{10.5} M_{\odot}$, and $M_{*}>10^{10.5} M_{\odot}$ )

for either centrals or satellites. The flat dependence on the halo mass for satellites suggests that the ram pressure stripping may not be the most dominant, or at least not the only channel, to suppress the star formation of massive satellites in groups and clusters. However, we note that the dividing line (50\% insideout and 50\% outside-in) adopted here is closer to the pure outside-in trajectory (dotted line) and hence it is possible that our results are affected by the small statistics of the outside-in galaxies. To test the robustness of our results, we also repeat the analyses using the other two dividing lines that correspond to the $60 \%$ inside-out versus $40 \%$ outside-in and $70 \%$ inside-out versus $30 \%$ outside-in contributions (see the Appendix). We find that the flat dependence of the outside-in fraction on the halo mass still holds in these two cases, despite the fraction of outside-in quenching galaxies increasing as a result of moving the dividing line away from the pure outside-in trajectory,

\section{Discussion}

\subsection{What Drives the Inside-out Quenching?}

Our results show that the fraction of galaxies showing an inside-out quenching pattern, strongly depends on the halo mass, regardless of being a central galaxy or a satellite. The increasing frequency of inside-out quenching with halo mass still holds even if we split the satellites into two stellar-mass bins. Furthermore, we find that more massive galaxies tend to have higher fractions of inside-out quenching than less massive ones, irrespective of their environments. This is in line with the finding in the literature that high-mass galaxies tend to exhibit suppressed sSFR in the galactic cores as opposed to low-mass galaxies (Belfiore et al. 2018; Liu et al. 2018; Sánchez et al. 2018). Our results suggest that the effect of inside-out quenching depends on both stellar mass and halo mass.

Among all the mechanisms that may suppress the star formation, AGN feedback (e.g., Bower et al. 2006; Croton et al. 2006; Fabian 2012) or morphological quenching (Martig et al. 2009) may potentially drive the features of the inside-out quenching. The study of the molecular gas properties of a preliminary set of MaNGA-selected green valley galaxies suggests that the gas is depleted in an inside-out fashion that can be possibly attributed to the AGN feedback (Lin et al. 2017a). In order to investigate whether these processes are responsible for the halo mass dependence of the inside-out quenching, we first investigate the frequency of AGNs and galaxies with high Sérsic index as a function of halo mass in our sample. For the study of AGNs, we utilize the emissionline-selected AGN candidates identified by Sánchez et al. (2018), updated with the MPL-6 version. The AGN sample 

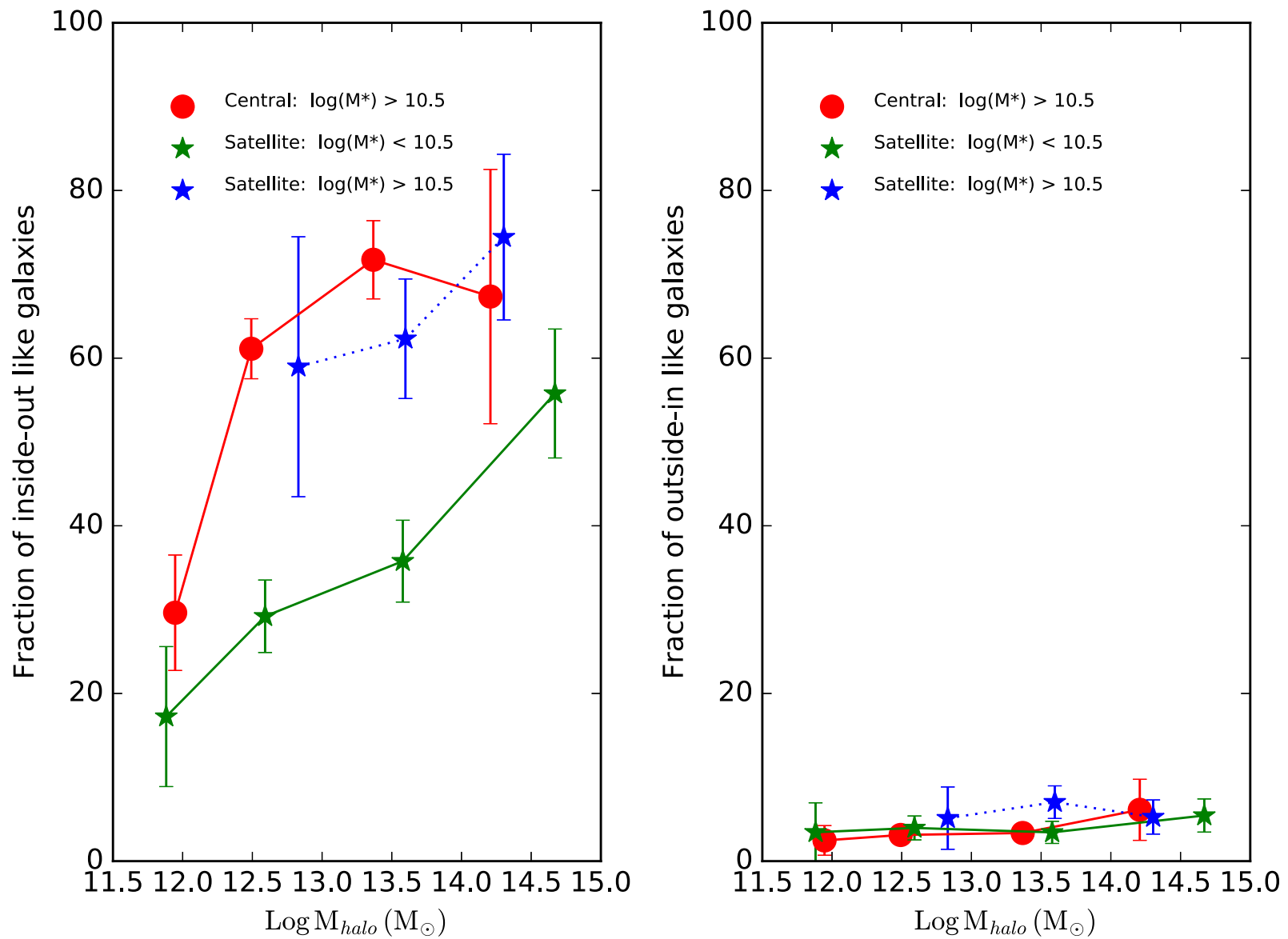

Figure 10. Fraction of inside-out-like (left panel) and outside-in-like (right panel) galaxies of centrals with $M_{*}>10^{10.5} M_{\odot}\left(\right.$ red symbols), satellites with $M_{*}<10^{10.5}$ $M_{\odot}$ (green symbols), and satellites with $M_{*}>10^{10.5} M_{\odot}$ (blue symbols). The dotted and solid lines correspond to high-mass and low-mass galaxies, respectively. The error bars are computed as the rms normalized by the square root of the sample size in each bin.

Table 1

Inside-out and Outside-in Quenching Fractions as a Function of Halo Mass

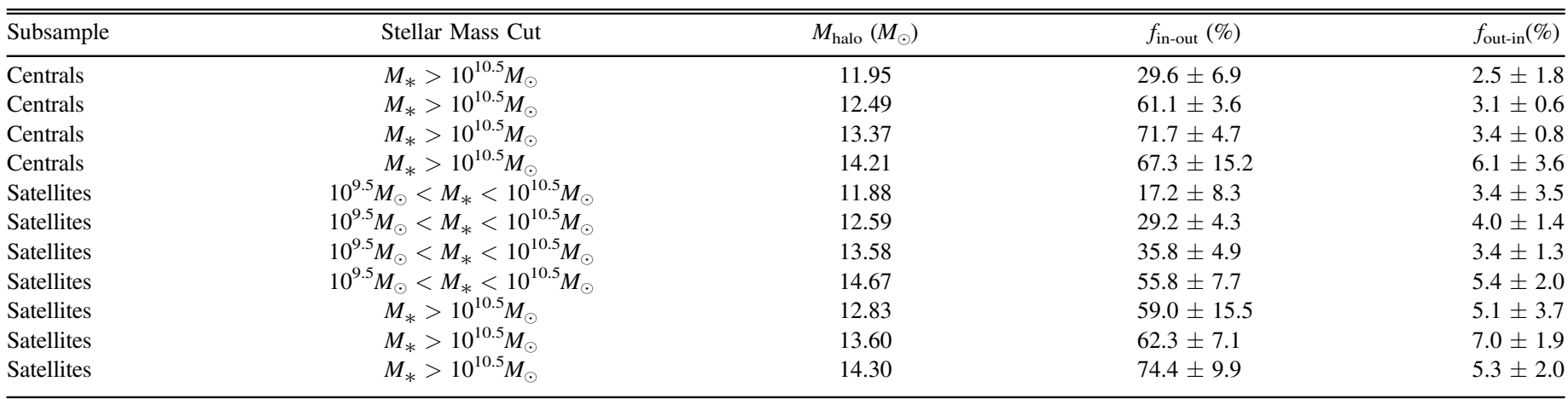

contains both Type-I and Type-II AGNs. It is found that the properties of their host galaxies differ slightly in the SFR versus stellar-mass plane-Type-I AGN hosts span a wide range of sSFR, from star-forming to quiescent, whereas Type-II AGN hosts are preferentially located in the green valley and the highsSFR end of the quiescent population (see Figure 4 of Sánchez et al. 2018, for details). Figure 11 shows the distributions of two types of AGNs on the $F_{q}$ versus $C_{q}$ plane. It is clear that Type-I and Type-II AGNs are distributed differently in this diagram-38\% and $15 \%$ of galaxies hosting a Type-I AGN show the inside-out and outside-in quenching patterns, respectively, compared to Type-II AGNs (63\% versus 5\%).
However, the location of Type-I AGN could be strongly affected by the lack of LI(N)ER detection in the central regions that are heavily ionized by the AGN itself, leading to the deficit of inside-out quenching Type-I AGN seen in the analysis.

In Figure 12 and Table 2 we show the ratio of the number of Type-I plus Type-II AGNs to the number of total galaxies with $M_{*}>10^{9.5} M_{\odot}$ as a function of halo mass. It can be seen that the AGN fraction peaks in halos with masses between $10^{12.5}$ and $10^{13.5} M_{\odot}$, roughly corresponding to the group scales, and drops toward massive cluster-scale halos. This trend remains similar if we restrict the sample to Type-I or Type-II AGNs only, and is different from the increasing inside-out fraction 


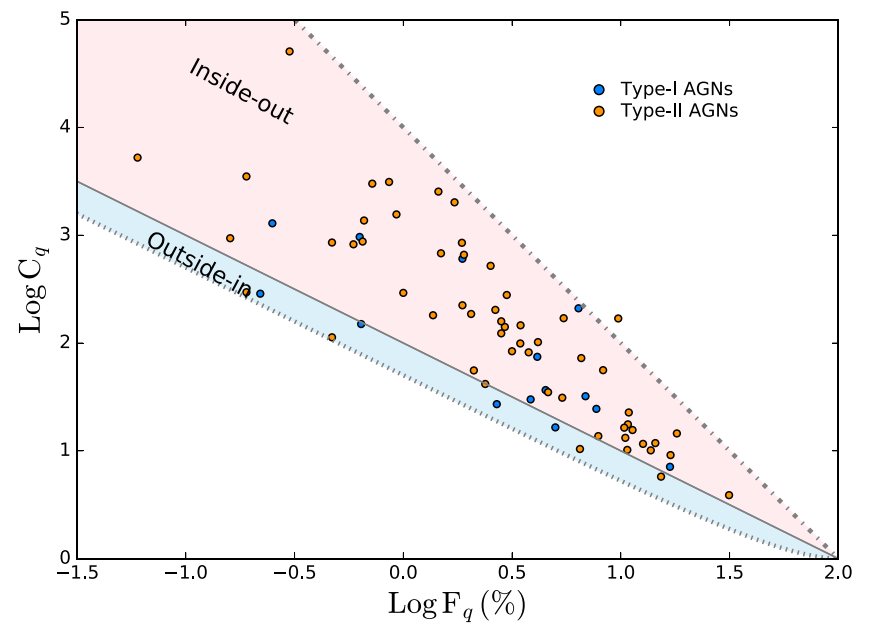

Figure 11. Distributions of MaNGA-selected AGN hosts on the quiescence $\left(F_{q}\right)$ vs. quenching concentration $\left(C_{q}\right)$ plane. The Type-I and type-II AGNs are shown in blue and orange, respectively. Similar to Figure 2, the light blue shaded area denotes the outside-in-like quenching mode, whereas the pink shaded region denotes the inside-out-like quenching mode.

Table 2

Fractions of AGNs and Galaxies with High Sérsic Index as a Function of Halo Mass

\begin{tabular}{lccc}
\hline \hline Subsample & Stellar Mass Cut & $M_{\text {halo }}\left(M_{\odot}\right)$ & Fraction(\%) \\
\hline AGN & $M_{*}>10^{9.5} M_{\odot}$ & 11.86 & $1.3 \pm 0.6$ \\
AGN & $M_{*}>10^{9.5} M_{\odot}$ & 12.46 & $3.8 \pm 0.6$ \\
AGN & $M_{*}>10^{9.5} M_{\odot}$ & 13.41 & $4.6 \pm 0.7$ \\
AGN & $M_{*}>10^{9.5} M_{\odot}$ & 14.53 & $0.9 \pm 0.5$ \\
$n \geqslant 3$ & $10^{9.5} M_{\odot}<M_{*}<10^{10.5} M_{\odot}$ & 11.89 & $31.0 \pm 11.8$ \\
$n \geqslant 3$ & $10^{9.5} M_{\odot}<M_{*}<10^{10.5} M_{\odot}$ & 12.61 & $36.1 \pm 4.9$ \\
$n \geqslant 3$ & $10^{9.5} M_{\odot}<M_{*}<10^{10.5} M_{\odot}$ & 13.60 & $41.2 \pm 5.3$ \\
$n \geqslant 3$ & $10^{9.5} M_{\odot}<M_{*}<10^{10.5} M_{\odot}$ & 14.76 & $49.7 \pm 7.1$ \\
$n \geqslant 3$ & $M_{*}>10^{10.5} M_{\odot}$ & 12.84 & $64.1 \pm 16.4$ \\
$n \geqslant 3$ & $M_{*}>10^{10.5} M_{\odot}$ & 13.61 & $76.9 \pm 8.3$ \\
$n \geqslant 3$ & $M_{*}>10^{10.5} M_{\odot}$ & 14.30 & $81.2 \pm 10.5$ \\
\hline
\end{tabular}

with halo mass. Our finding that the frequency of optically selected AGNs peaks on group scales is similar to the trend found for samples of X-ray-selected and radio-selected AGNs (Sánchez \& González-Serrano 2002; Best 2004; Arnold et al. 2009; Davies et al. 2017).

Figure 13 shows how galaxies with different Sérsic indices $(n)$ are distributed in the $F_{q}$ and $C_{q}$ plane. We see that galaxies with higher Sérsic index tend to occupy the inside-out regions; $70 \%$ and $6 \%$ of galaxies with $n>3$ are classified as inside-out and outside-in quenching, respectively. On the other hand, only $17 \%$ and $1 \%$ of galaxies with $n<3$ are classified as inside-out and outside-in quenching, respectively. The high inside-out quenching fraction seen in galaxies with high $n$ is somewhat expected since it is well-known that galaxies with high Sérsic index or bulge-to-total (B/T) have lower sSFR (Whitaker et al. 2015; Pan et al. 2018) and that the bulges tend to have old stellar populations (González Delgado et al. 2015; McDermid et al. 2015; López Fernández et al. 2018) and lower sSFR (Pan et al. 2018). In Figure 12 (also see Table 2) we show the fraction of satellite galaxies with $n>3$ as a function of halo mass for two stellar-mass bins (red symbols). In the case of the low-mass satellites for which we have enough statistics, it is shown that the fraction of high Sérsic index galaxies strongly increases with halo mass, as seen in the fraction of inside-out galaxies. The

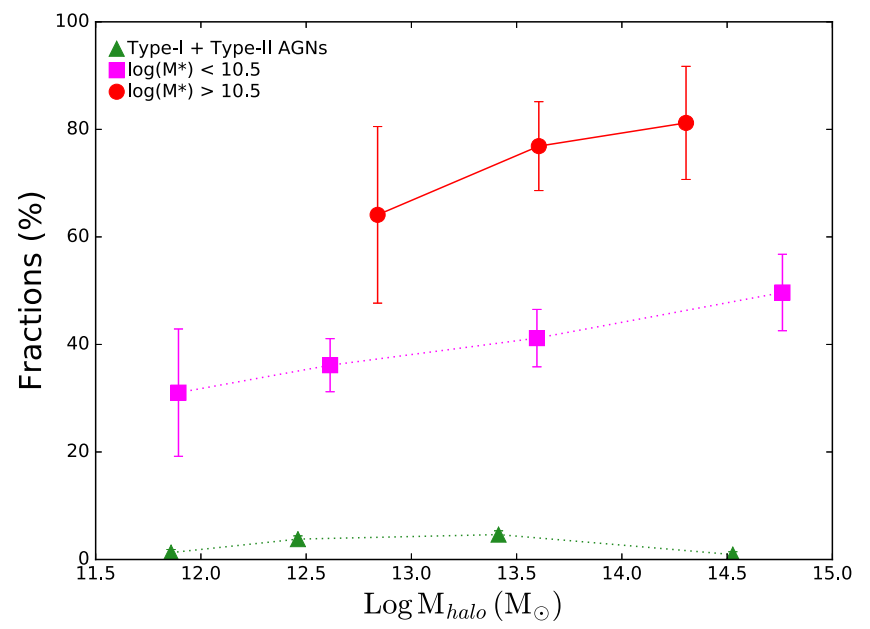

Figure 12. Fraction of type-I plus type-II AGNs (green triangles), high Sérsic galaxies with $M_{*}<10^{10.5} M_{\odot}$ (magenta symbols), and high Sérsic galaxies with $M_{*}>10^{10.5} M_{\odot}$ (red symbols) as a function of halo mass. The error bars are computed as the rms normalized by the square root of the sample size in each bin.

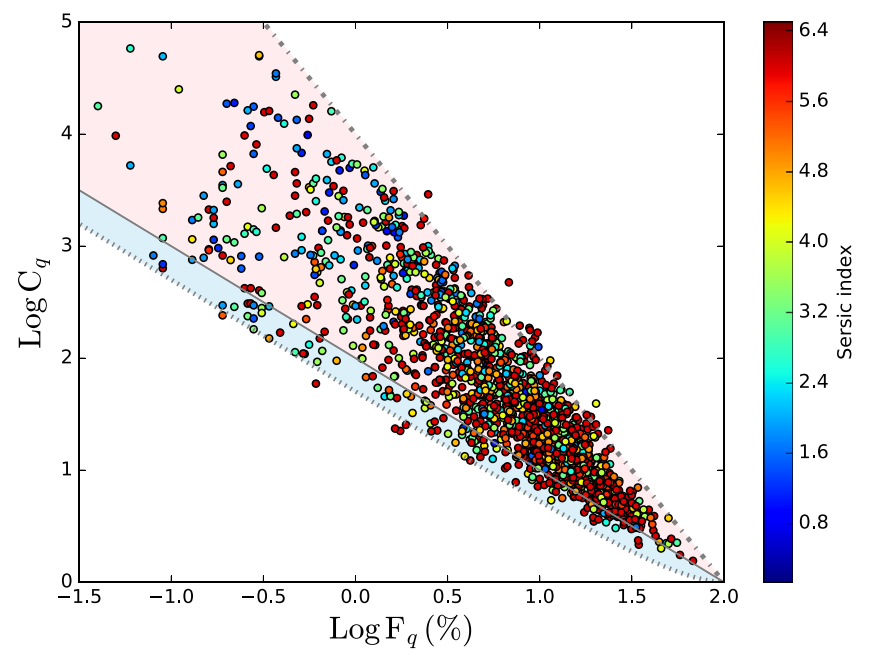

Figure 13. Distributions of MaNGA galaxies on the quiescence $\left(F_{q}\right)$ vs quenching concentration $\left(C_{q}\right)$ plane, color-coded according to Sérsic index. Similar to Figure 2, the light blue shaded area denotes the outside-in-like quenching mode whereas the pink shaded region denotes the inside-out-like quenching mode.

trend remains similar if we change the threshold of the Sérsic index to a higher value of 3.5 or a lower value of 2.5.

Unlike the AGN fraction, which is much smaller compared to the inside-out fraction, the fraction of galaxies with high Sérsic index is comparable to that of the inside-out galaxies. The good agreement in the halo mass dependence between the high Sérsic fraction and inside-out fraction indicates that morphological quenching could be responsible for the growing inside-out quenched galaxies found in more massive halos. Our results seem to favor morphological quenching (Martig et al. 2009) over AGN feedback as the primary cause for the similar environment dependence of the inside-out quenching. However, it should be noted that the duty cycles of AGNs and the timescale of morphological quenching may be quite different. As discussed in Sánchez et al. (2018), the timescale of the active AGN phase may be on the order of $\sim 0.1$ Gyr (Parma et al. 2007; Shulevski et al. 2015), which could be shorter than the star formation quenching timescale itself. On the other 
hand, morphological quenching operates over a longer period once the bulge component forms. Galaxies showing inside-out quenching features may be a result of accumulated quenching events over time in the past rather than an ongoing event. Therefore, we cannot rule out the possibility that galaxies are quenched when they are located in groups (the "preprocessing" effect) where the AGN is more frequent, as seen in our data, after which they fall into bigger clusters.

An alternative process that is commonly thought to operate in dense environments, called "strangulation" (or "starvation"), refers to the situation where galaxies fail to replenish the gas due to the removal of an extended gas halo. This effect is suggested to be stronger when galaxies fall into dense environments such as groups or cluster of galaxies (Larson et al. 1980; Kawata \& Mulchaey 2008). Strangulation is predicted to suppress the star formation uniformly over the entire galaxy and therefore produces a spatial distribution of star formation that is distinct from other mechanisms. It has been argued that strangulation is the primary channel to quench the star formation of satellites (van den Bosch et al. 2008; Peng et al. 2015; Spindler et al. 2018), although the exact dependence of environments has not been well constrained. There has been an increasing number of studies finding the global suppression of star formation in green valley galaxies or satellite galaxies by comparing their star formation rate or sSFR gradients with respect to the reference sample (Belfiore et al. 2018; Spindler et al. 2018), favoring the strangulation quenching scenario. However, it should be stressed that spatially uniform suppression of star formation is not necessarily in contradiction with the inside-out and outside-in quenching defined in this work, which are characterized based on the already quenched areas within the galaxies. As the areas with sSFR below a certain threshold will become a LI(N)ER and hence are defined as quenched regions first, depending on the initial slope of the star formation rate profile, the global reduction of star formation may result in inside-out or outside-in quenching features.

\subsection{What Drives the Outside-in Quenching?}

A few processes associated with environments may potentially be responsible for the outside-in quenching features. Ram pressure stripping has long been suggested to be one of the primary mechanisms that suppress the star formation of galaxies in groups and clusters. When galaxies fall into massive groups or clusters, they experience winds because of the relative motion of the hot intraclustser medium (ICM). As a result, the diffuse interstellar medium (IGM) in the outer parts of galaxies can be stripped, leading to the cessation of star formation. An alternative explanation for outside-in quenching is through galaxy mergers. Both observations and simulations have demonstrated that galaxy interactions can induce gas inflow triggering central starbursts (Barnes \& Hernquist 1996; Cox et al. 2006; Lin et al. 2007; but see Bergvall et al. 2003; Barrera-Ballesteros et al. 2015; Fensch et al. 2017 for different findings) and possibly AGN activity (Di Matteo et al. 2005; Hopkins et al. 2006; Ellison et al. 2008). Recent merger simulations suggest that the star formation could be suppressed at large galactocentric radii during galaxy-galaxy interactions as a result of gas inflow, while the star formation rate in the central part of galaxies is strongly enhanced, followed by an immediate truncation of star formation after the gas fuel is fully consumed. (Moreno et al. 2015). Under these circumstances, quenching may possibly occur in an outside-in fashion during some phases of galaxy mergers. Observations and simulations found that galaxy mergers are typically more frequent in dense environments (Lin et al. 2010) and peaks in halos with halo mass $\sim 10^{13} M_{\odot}($ Jian et al. 2012). Therefore, if the ram pressure stripping process is effective at removing the cold gas from the outskirts of galaxies, we expect to see a higher fraction of galaxies with outside-in patterns in massive halos, especially in clusters of galaxies. On the other hand, if galaxygalaxy interactions dominate the outside-in quenching, the fraction of outside-in quenching galaxies is expected to peak around group scales.

As shown in Figure 10, the dependence of $f_{\text {out-in }}$ on halo mass is nearly flat for both low-mass and high-mass satellites, which indicates that neither ram pressure stripping nor galaxy interactions play a dominant role in producing the outside-in quenching processes. Either both processes may contribute to some degrees or there could be some other mechanism that causes the outside-in quenching features. Nevertheless, it is worth noting that the majority of (partially) quenched satellite galaxies is instead in the inside-out quenching category, which strongly depends on halo mass. In other words, while ram pressure stripping or galaxy interactions may take place in massive halos, they may not be the primary mechanisms that produce a higher fraction of passive galaxies seen in groups and clusters relative to the field galaxies (Lin et al. 2014; Jian et al. 2017, 2018).

\subsection{Comparisons with Other Works}

Since the advent of large IFU surveys, there has been a growing number of environmental studies based on the spatial distributions of stellar populations and star formation rate (e.g., Goddard et al. 2017; Schaefer et al. 2017; Zheng et al. 2017; Medling et al. 2018; Spindler et al. 2018). While most studies are based on gradients of age, star formation rate, or sSFR, our approach probes the spatial distribution of quenched areas, which provides complementary information. Therefore, it would be intriguing to compare our results with previous environmental works based on the IFU observations.

Spindler et al. (2018) studied the environmental effects by exploring the sSFR gradients in central and satellite galaxies using 1494 MaNGA galaxies. In their study, they classified their galaxies into centrally unsuppressed and suppressed types. The former class on average shows flat profiles in SSFR, while the latter exhibits positive slopes in the sSFR gradient. By comparing the sSFR of the satellites relative to the centrals with the same stellar mass, they found a global suppression in the satellites and concluded that their results favor the strangulation scenario. Supposedly, if the reduction in the sSFR is spatially uniform, the centrally suppressed galaxies will display the inside-out quenching feature according to our definition, as the central spaxels become LI(N)ER first. In contrast, the centrally unsuppressed galaxies will not appear as inside-out or outsidein quenching because the local spaxels become the LI(N)ER regions simultaneously once their sSFRs drop below the $\mathrm{H}$ II threshold. Although it is not straightforward to directly translate our results to the gradient studies given the different nature of our approaches, both works seem to indicate that outside-in quenching plays a less vital role in environmental quenching.

Nevertheless, the greater fraction of inside-out quenching over outside-in quenching found in our work seems to be in direct conflict with the work carried out by Schaefer et al. (2017), who studied the star formation rate gradients in the SAMI sample and concluded that in dense environments the star formation quenches outside-in. However, there is a 
fundamental difference between our analyses: the environments are traced by the local nearest neighbor density in Schaefer et al. (2017), whereas in this work we focus on the halo mass. These two environment proxies do not necessarily have a oneto-one correspondence. As shown in Figure 12 of Lin et al. (2016), there is a wide spread of local density at a given halo mass-at low redshifts the highest density is actually dominated by groups rather than clusters. Thus, the relative strength of various environment effects may differ between our samples.

\section{Conclusions}

Using the spatially resolved datacubes of 2915 galaxies drawn from SDSS-IV MaNGA, we study the quenching properties of galaxies as a function of halo mass in order to probe environmental quenching effects. We use LI(N)ER regions with low $\mathrm{EW}(\mathrm{H} \alpha)$ to trace the quenched areas and we define two non-parametric parameters, quiescence $\left(F_{q}\right)$ and its concentration $\left(C_{q}\right)$, to quantify the strength and the spatial distribution of the quenched areas. With the combination of these two parameters, we are able to classify galaxies into two categories, inside-out and outside-in quenching, and to study their frequency in different masses of halos. Our results can be summarized as follows:

1. The fraction of galaxies showing inside-out quenching increases with both stellar mass (at a fixed halo mass) and halo mass (at a fixed stellar mass). On the other hand, the frequency of outside-in quenching is almost independent of the halo mass. In nearly all local environments, the frequency of inside-out quenching is higher than the frequency of outside-in quenching at a fixed stellar mass and halo mass. The difference between the two quenching modes is more pronounced for galaxies located in the more massive halos. Our results suggest that inside-out quenching is the dominant quenching mode in all environments.

2. We find that the increasing fraction of galaxies exhibiting a high Sérsic index (and hence a greater bulge component) with halo mass is similar to the halo mass dependence of the inside-out quenching, suggesting a plausible link between these two phenomena. On the other hand, the frequency of AGNs peaks at group scales, differing from the rising curve of the inside-out quenching with respect to the halo mass. Our result seems to favor the morphological quenching over AGN feedback as a primary mechanism driving the environmental dependence of the inside-out quenching, although this could be affected by the issue of uncertainties in the AGN duty cycle.

3. The lack of halo mass dependence of outside-in quenching suggests that neither ram pressure stripping nor a galaxy-galaxy merger is the dominant process in massive halos. It is likely that both mechanisms contribute to the outside-in quenching seen in different environments.

Our method characterizes the quenched areas and provides a complementary approach to investigating the quenching mechanisms with respect to other studies that are based on the spatial gradients in the sSFR and stellar populations. This study has revealed that both inside-out and outside-in quenching coexist in different environments and that insideout quenching dominates in the more massive halos.
Combining MaNGA data with future spatially resolved molecular gas observations from ALMA will be key to further understanding the cause of quenching in different environments.

We thank the anonymous referee for useful suggestions that improved the clarity of this paper. The work is supported by the Academia Sinica under the Career Development Award CDA107-M03 and the Ministry of Science \& Technology of Taiwan under the grant MOST 107-2119-M-001-024. L. Lin acknowledges H. Yee for his useful suggestions to this work. S.F.S. is grateful for the support of a CONACYT (Mexico) grant CB285080, and funding from the PAPIIT-DGAPA-IA101217 (UNAM). M.A.F. is grateful for financial support from the CONICYT Astronomy Program CAS-CONICYT project No. CAS17002, sponsored by the Chinese Academy of Sciences (CAS), through a grant to the CAS South America Center for Astronomy (CASSACA) in Santiago, Chile. I.L. acknowledges partial financial support from PROYECTO FONDECYT REGULAR 1150345. R.R. thanks FAPERGS and CNPq for financial support.

Funding for the Sloan Digital Sky Survey IV has been provided by the Alfred P. Sloan Foundation, the U.S. Department of Energy Office of Science, and the Participating Institutions. SDSS-IV acknowledges support and resources from the Center for High-Performance Computing at the University of Utah. The SDSS website is www.sdss.org. SDSS-IV is managed by the Astrophysical Research Consortium for the Participating Institutions of the SDSS Collaboration including the Brazilian Participation Group, the Carnegie Institution for Science, Carnegie Mellon University, the Chilean Participation Group, the French Participation Group, Harvard-Smithsonian Center for Astrophysics, Instituto de Astrofísica de Canarias, The Johns Hopkins University, Kavli Institute for the Physics and Mathematics of the Universe (IPMU)/University of Tokyo, Lawrence Berkeley National Laboratory, Leibniz Institut für Astrophysik Potsdam (AIP), Max-Planck-Institut für Astronomie (MPIA Heidelberg), Max-Planck-Institut für Astrophysik (MPA Garching), MaxPlanck-Institut für Extraterrestrische Physik (MPE), National Astronomical Observatory of China, New Mexico State University, New York University, University of Notre Dame, Observatário Nacional/MCTI, The Ohio State University, Pennsylvania State University, Shanghai Astronomical Observatory, United Kingdom Participation Group, Universidad Nacional Autónoma de México, University of Arizona, University of Colorado Boulder, University of Oxford, University of Portsmouth, University of Utah, University of Virginia, University of Washington, University of Wisconsin, Vanderbilt University, and Yale University.

\section{Appendix \\ Toy Models}

Let us consider a perfectly circular galaxy with external radius $R$. The inside-out quenching region is a circular section of the galaxy with a radius $R_{1}$ around the nucleus. The outsidein quenching region extends from radius $R_{2}$ to $R$. We will define $R \equiv 1$ so that $R_{1}$ and $R_{2}$ are numerically equivalent to fractions of the total galaxy radius. In that case, the total circular area of a galaxy is numerically identical to $\pi$.

The quenched fraction $F_{q}$ of a "mixed" galaxy (i.e., presenting both inside-out and outside-in quenching) is given 


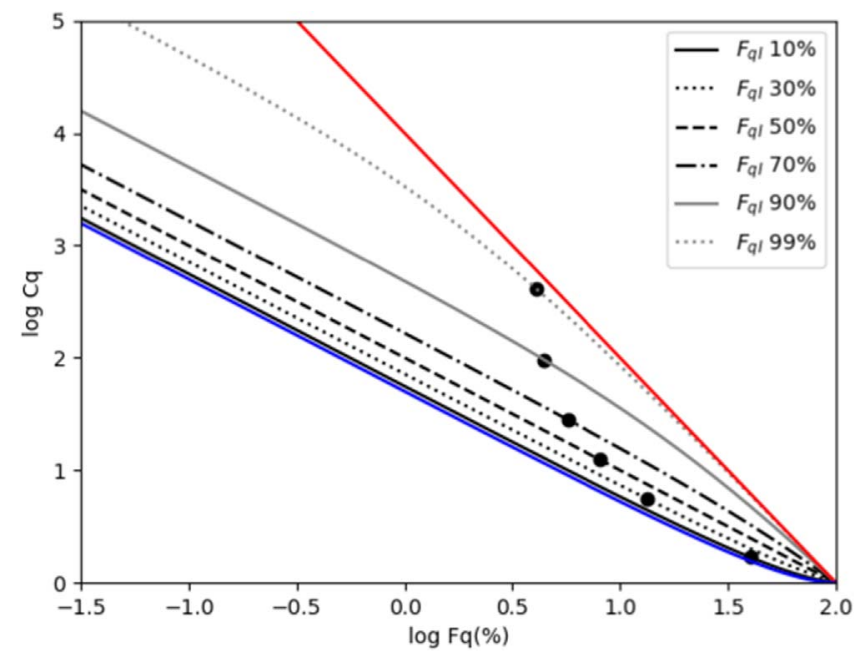

Figure 14. Distributions of toy models showing various mixing of inside-out and outside-in quenching on the quiescence $\left(F_{q}\right)$ vs. quenching concentration $\left(C_{q}\right)$ plane using Equation (23). The red and blue lines denote the pure insideout and outside-in quenching lines, respectively. The black lines show the model lines with different fractions of inside-out contributions $\left(F_{q}\right.$, increasing from the bottom to the top. The dashed line, corresponding to the 50\% insideout and 50\% outside-in quenching, is used to separate the two modes of quenching in this work. The black circles show the position of a galaxy with inside-out quenching radius equal to $20 \%$ of the total galaxy radius.

by the fraction of quenched pixels to the total galaxy area, i.e.,

$$
\begin{gathered}
F_{q}=\frac{\text { internal quenching }+ \text { external quenching }}{\pi}, \\
=\frac{\pi R_{1}^{2}+\left(\pi-\pi R_{2}^{2}\right)}{\pi}, \\
=1+R_{1}^{2}-R_{2}^{2} .
\end{gathered}
$$

The contribution of inside-out quenching to the $F_{q}$ value above, $F_{q I}$, can be expressed as

$$
\begin{gathered}
F_{q I}=\frac{\pi R_{1}^{2}}{\pi R_{1}^{2}+\left(\pi-\pi R_{2}^{2}\right)}, \\
=\frac{R_{1}^{2}}{F_{q}}
\end{gathered}
$$

so we can express $F_{q}$ in terms of $F_{q I}$ as

$$
F_{q}=\frac{R_{1}^{2}}{F_{q I}}
$$

The concentration $C_{q}$ is defined as

$$
C_{q}=\frac{\sum r_{\text {all }}^{2}}{\sum r_{\text {quenched }}^{2}}
$$

Expressing the density of pixels as $\rho$ and considering the infinitesimal limit, the squared summation of radial distances $r$ up to a radius $R$ can be written as

$$
\begin{aligned}
\sum r^{2} & =\int_{0}^{R} \rho 2 \pi r^{3} d r, \\
& =\frac{\pi \rho R^{4}}{2},
\end{aligned}
$$

so we can express the concentration of a "mixed" galaxy as

$$
\begin{gathered}
C_{q}=\frac{\pi \rho / 2}{\pi \rho R_{1}^{4} / 2+\pi \rho\left(1-R_{2}^{4}\right) / 2}, \\
=\frac{1}{1+R_{1}^{4}-R_{2}^{4}},
\end{gathered}
$$

where $\pi \rho / 2$ is the result of $\sum r_{\text {all }}^{2}$. From Equation (14),

$$
R_{1}^{4}=\left(F_{q} F_{q I}\right)^{2},
$$

and from Equation (11),

$$
R_{2}^{4}=\left(1+R_{1}^{2}-F_{q}\right)^{2},
$$

so we can rewrite the solution for $C_{q}$ (Equation (18)) as

$$
\begin{aligned}
& C_{q}=\frac{1}{1+\left(F_{q} F_{q I}\right)^{2}-\left(1+R_{1}^{2}-F_{q}\right)^{2}}, \\
& =\frac{1}{1+\left(F_{q} F_{q I}\right)^{2}-\left(1+F_{q} F_{q I}-F_{q}\right)^{2}} .
\end{aligned}
$$

Now, in order to express $F_{q}$ and $C_{q}$ in the same notation as in the paper (log scale, and $F_{q}$ as a percent) we define

$$
\begin{gathered}
x=\log _{10} 100 \times F_{q}, \\
y=\log _{10} C_{q},
\end{gathered}
$$

so that the loci of constant $F_{q I}$ values are given by

$$
y=\log _{10} \frac{1}{1+\left(F_{q I} 10^{x} / 100\right)^{2}-\left(1+F_{q I} 10^{x} / 100-10^{x} / 100\right)^{2}} .
$$

Note that the $F_{q I}$ values are fractions of the quenched area, not percent values.

\section{ORCID iDs}

Lihwai Lin (1) https://orcid.org/0000-0001-7218-7407

Bau-Ching Hsieh (1) https://orcid.org/0000-0001-5615-4904

Hsi-An Pan (i) https://orcid.org/0000-0002-1370-6964

Dmitry Bizyaev (i) https://orcid.org/0000-0002-3601-133X

Ivan Lacerna (i) https://orcid.org/0000-0002-7802-7356

Yu Rong (i) https://orcid.org/0000-0002-2204-6558

Niv Drory i https://orcid.org/0000-0002-7339-3170

\section{References}

Abdurro'uf, A., \& Masayuki 2018, MNRAS, 479, 5083

Albareti, F. D., Allende Prieto, C., Almeida, A., et al. 2017, ApJS, 233, 25

Argudo-Fernández, M., Lacerna, I., \& Duarte Puertas, S. 2018, A\&A, 620, A113

Arnold, T. J., Martini, P., Mulchaey, J. S., Berti, A., \& Jeltema, T. E. 2009 , ApJ, 707, 1691

Baldry, I. K., Balogh, M. L., Bower, R. G., et al. 2006, MNRAS, 373, 469

Baldry, I. K., Glazebrook, K., Brinkmann, J., et al. 2004, ApJ, 600, 681

Baldwin, J. A., Phillips, M. M., \& Terlevich, R. 1981, PASP, 93, 5

Barnes, J. E., \& Hernquist, L. 1996, ApJ, 471, 115

Barrera-Ballesteros, J. K., Sánchez, S. F., García-Lorenzo, B., et al. 2015, A\&A, 579, A45

Belfiore, F., Maiolino, R., Bundy, K., et al. 2018, MNRAS, 477, 3014

Belfiore, F., Maiolino, R., Maraston, C., et al. 2016, MNRAS, 461, 3111

Belfiore, F., Maiolino, R., Tremonti, C., et al. 2017, MNRAS, 469, 151

Bergvall, N., Laurikainen, E., \& Aalto, S. 2003, A\&A, 405, 31

Best, P. N. 2004, MNRAS, 351, 70

Binette, L., Magris, C. G., Stasińska, G., \& Bruzual, A. G. 1994, A\&A, 292, 13

Blanton, M. R., Bershady, M. A., Abolfathi, B., et al. 2017, AJ, 154, 28

Blanton, M. R., \& Moustakas, J. 2009, ARA\&A, 47, 159

Blanton, M. R., Schlegel, D. J., Strauss, M. A., et al. 2005, AJ, 129, 2562 
Bower, R. G., Benson, A. J., Malbon, R., et al. 2006, MNRAS, 370, 645 Brown, T., Catinella, B., Cortese, L., et al. 2017, MNRAS, 466, 1275 Bryant, J. J., Owers, M. S., Robotham, A. S. G., et al. 2015, MNRAS, 447, 2857

Bundy, K., Bershady, M. A., Law, D. R., et al. 2015, ApJ, 798, 7

Calzetti, D. 2001, PASP, 113, 1449

Cano-Díaz, M., Sánchez, S. F., Zibetti, S., et al. 2016, ApJL, 821, L26

Cid Fernandes, R., Pérez, E., García Benito, R., et al. 2013, A\&A, 557, A86

Cid Fernandes, R., Stasińska, G., Mateus, A., \& Vale Asari, N. 2011, MNRAS, 413, 1687

Cid Fernandes, R., Stasińska, G., Schlickmann, M. S., et al. 2010, MNRAS, 403, 1036

Cooper, M. C., Newman, J. A., Coil, A. L., et al. 2007, MNRAS, 376, 1445

Cox, T. J., Jonsson, P., Primack, J. R., \& Somerville, R. S. 2006, MNRAS, 373, 1013

Croton, D. J., Springel, V., White, S. D. M., et al. 2006, MNRAS, 365, 11

Davies, R. I., Hicks, E. K. S., Erwin, P., et al. 2017, MNRAS, 466, 4917

Di Matteo, T., Springel, V., \& Hernquist, L. 2005, Natur, 433, 604

Dressler, A. 1980, ApJ, 236, 351

Drory, N., MacDonald, N., Bershady, M. A., et al. 2015, AJ, 149, 77

Ellison, S. L., Patton, D. R., Simard, L., \& McConnachie, A. W. 2008, AJ, 135, 1877

Ellison, S. L., Sánchez, S. F., Ibarra-Medel, H., et al. 2018, MNRAS, 474, 2039

Fabian, A. C. 2012, ARA\&A, 50, 455

Falcón-Barroso, J., Sánchez-Blázquez, P., Vazdekis, A., et al. 2011, A\&A, 532, A95

Fensch, J., Renaud, F., Bournaud, F., et al. 2017, MNRAS, 465, 1934

Fischera, J., \& Dopita, M. 2005, ApJ, 619, 340

Fritz, J., Moretti, A., Gullieuszik, M., et al. 2017, ApJ, 848, 132

Goddard, D., Thomas, D., Maraston, C., et al. 2017, MNRAS, 465, 688

Gomes, J. M., Papaderos, P., Kehrig, C., et al. 2016, A\&A, 588, A68

González Delgado, R. M., Cid Fernandes, R., Pérez, E., et al. 2016, A\&A, 590, A44

González Delgado, R. M., García-Benito, R., Pérez, E., et al. 2015, A\&A, 581, A103

González Delgado, R. M., Pérez, E., Cid Fernandes, R., et al. 2014, A\&A, 562, A47

González Delgado, R. M., Pérez, E., Cid Fernandes, R., et al. 2017, A\&A, 607, A128

Gunn, J. E., \& Gott, J. R. I. 1972, ApJ, 176, 1

Gunn, J. E., Siegmund, W. A., Mannery, E. J., et al. 2006, AJ, 131, 2332

Haines, C. P., Pereira, M. J., Smith, G. P., et al. 2013, ApJ, 775, 126

Hopkins, P. F., Hernquist, L., Cox, T. J., et al. 2006, ApJS, 163, 1

Hsieh, B. C., Lin, L., Lin, J. H., et al. 2017, ApJL, 851, L24

Ibarra-Medel, H. J., Sánchez, S. F., Avila-Reese, V., et al. 2016, MNRAS, 463, 2799

Jian, H.-Y., Lin, L., \& Chiueh, T. 2012, ApJ, 754, 26

Jian, H.-Y., Lin, L., Lin, K.-Y., et al. 2017, ApJ, 845, 74

Jian, H.-Y., Lin, L., Oguri, M., et al. 2018, PASJ, 70, S23

Kauffmann, G. 1996, MNRAS, 281, 487

Kauffmann, G., Heckman, T. M., White, S. D. M., et al. 2003, MNRAS, 341,33

Kawata, D., \& Mulchaey, J. S. 2008, ApJL, 672, L103

Kewley, L. J., Dopita, M. A., Sutherland, R. S., Heisler, C. A., \& Trevena, J. 2001, ApJ, 556, 121

Kewley, L. J., Groves, B., Kauffmann, G., \& Heckman, T. 2006, MNRAS, 372,961

Koyama, Y., Smail, I., Kurk, J., et al. 2013, MNRAS, 434, 423

Kuntschner, H., Smith, R. J., Colless, M., et al. 2002, MNRAS, 337, 172

Lacerna, I., Argudo-Fernández, M., \& Duarte Puertas, S. 2018, A\&A, 620, A117

Lacerna, I., Hernández-Toledo, H. M., Avila-Reese, V., Abonza-Sane, J., \& del Olmo, A. 2016, A\&A, 588, A79

Larson, R. B., Tinsley, B. M., \& Caldwell, C. N. 1980, ApJ, 237, 692

Law, D. R., Yan, R., Bershady, M. A., et al. 2015, AJ, 150, 19

Li, C., Wang, E., Lin, L., et al. 2015, ApJ, 804, 125

Lin, L., Belfiore, F., Pan, H.-A., et al. 2017a, ApJ, 851, 18
Lin, L., Capak, P. L., Laigle, C., et al. 2016, ApJ, 817, 97

Lin, L., Cooper, M. C., Jian, H.-Y., et al. 2010, ApJ, 718, 1158

Lin, L., Jian, H.-Y., Foucaud, S., et al. 2014, ApJ, 782, 33

Lin, L., Koo, D. C., Weiner, B. J., et al. 2007, ApJL, 660, L51

Lin, L., Lin, J.-H., Hsu, C.-H., et al. 2017b, ApJ, 837, 32

Liu, F. S., Jia, M., Yesuf, H. M., et al. 2018, ApJ, 860, 60

López Fernández, R., González Delgado, R. M., Pérez, E., et al. 2018, A\&A, 615, A27

Martig, M., Bournaud, F., Teyssier, R., \& Dekel, A. 2009, ApJ, 707, 250

Martins, L. P., González Delgado, R. M., Leitherer, C., Cerviño, M., \& Hauschildt, P. 2005, MNRAS, 358, 49

McCarthy, I. G., Frenk, C. S., Font, A. S., et al. 2008, MNRAS, 383, 593

McDermid, R. M., Alatalo, K., Blitz, L., et al. 2015, MNRAS, 448, 3484

Medling, A. M., Cortese, L., Croom, S. M., et al. 2018, MNRAS, 475, 5194

Mihos, J. C., \& Hernquist, L. 1994, ApJ, 431, L9

Moore, B., Katz, N., Lake, G., Dressler, A., \& Oemler, A, 1996, Natur, 379,613

Moreno, J., Torrey, P., Ellison, S. L., et al. 2015, MNRAS, 448, 1107

Muzzin, A., Wilson, G., Yee, H. K. C., et al. 2012, ApJ, 746, 188

Pan, H.-A., Lin, L., Hsieh, B.-C., et al. 2018, ApJ, 854, 159

Pan, Z., Li, J., Lin, W., et al. 2015, ApJL, 804, L42

Papaderos, P., Gomes, J. M., Vílchez, J. M., et al. 2013, A\&A, 555, L1

Parma, P., Murgia, M., de Ruiter, H. R., et al. 2007, A\&A, 470, 875

Peng, Y., Maiolino, R., \& Cochrane, R. 2015, Natur, 521, 192

Peng, Y.-j., Lilly, S. J., Kovač, K., et al. 2010, ApJ, 721, 193

Pérez, E., Cid Fernandes, R., González Delgado, R. M., et al. 2013, ApJL, 764, L1

Roberts, M. S., \& Haynes, M. P. 1994, ARA\&A, 32, 115

Sánchez, S. F., Avila-Reese, V., Hernandez-Toledo, H., et al. 2018, RMxAA, 54,217

Sánchez, S. F., \& González-Serrano, J. I. 2002, A\&A, 396, 773

Sánchez, S. F., Kennicutt, R. C., Gil de Paz, A., et al. 2012, A\&A, 538, A8

Sánchez, S. F., Pérez, E., Rosales-Ortega, F. F., et al. 2015, A\&A, 574, A47

Sánchez, S. F., Pérez, E., Sánchez-Blázquez, P., et al. 2016a, RMxAA, 52, 21

Sánchez, S. F., Pérez, E., Sánchez-Blázquez, P., et al. 2016b, RMxAA, 52 171

Sánchez, S. F., Rosales-Ortega, F. F., Jungwiert, B., et al. 2013, A\&A, 554, A58

Sánchez-Blázquez, P., Peletier, R. F., Jiménez-Vicente, J., et al. 2006 MNRAS, 371, 703

Sarzi, M., Shields, J. C., Schawinski, K., et al. 2010, MNRAS, 402, 2187

Schaefer, A. L., Croom, S. M., Allen, J. T., et al. 2017, MNRAS, 464, 121

Shulevski, A., Morganti, R., Barthel, P. D., et al. 2015, A\&A, 583, A89

Singh, R., van de Ven, G., Jahnke, K., et al. 2013, A\&A, 558, A43

Smee, S. A., Gunn, J. E., Uomoto, A., et al. 2013, AJ, 146, 32

Spindler, A., Wake, D., Belfiore, F., et al. 2018, MNRAS, 476, 580

Stasińska, G., Vale Asari, N., Cid Fernandes, R., et al. 2008, MNRAS, 391, L29

Tacchella, S., Carollo, C. M., Renzini, A., et al. 2015, Sci, 348, 314

Trager, S. C., Faber, S. M., \& Dressler, A. 2008, MNRAS, 386, 715

van den Bosch, F. C., Aquino, D., Yang, X., et al. 2008, MNRAS, 387, 79

Vazdekis, A., Sánchez-Blázquez, P., Falcón-Barroso, J., et al. 2010, MNRAS, 404, 1639

Veilleux, S., \& Osterbrock, D. E. 1987, ApJS, 63, 295

Vogt, F. P. A., Dopita, M. A., \& Kewley, L. J. 2013, ApJ, 768, 151

Vulcani, B., Poggianti, B. M., Finn, R. A., et al. 2010, ApJL, 710, L1

Wake, D. A., Bundy, K., Diamond-Stanic, A. M., et al. 2017, AJ, 154, 86

Wang, E., Li, C., Xiao, T., et al. 2018, ApJ, 856, 137

Wetzel, A. R., Tinker, J. L., \& Conroy, C. 2012, MNRAS, 424, 232

Whitaker, K. E., Franx, M., Bezanson, R., et al. 2015, ApJL, 811, L12

Yan, R., \& Blanton, M. R. 2012, ApJ, 747, 61

Yan, R., Bundy, K., Law, D. R., et al. 2016a, AJ, 152, 197

Yan, R., Tremonti, C., Bershady, M. A., et al. 2016b, AJ, 151, 8

Yang, X., Mo, H. J., van den Bosch, F. C., et al. 2007, ApJ, 671, 153

Yang, X., Mo, H. J., \& van den Bosch, F. C. 2008, ApJ, 676, 248

Zhang, K., Yan, R., Bundy, K., et al. 2017, MNRAS, 466, 3217

Zheng, Z., Wang, H., Ge, J., et al. 2017, MNRAS, 465, 4572 